\title{
Superconformal indices of generalized Argyres-Douglas theories from 2d TQFT
}

\author{
Jaewon Song \\ Department of Physics, University of California, San Diego, \\ 9500 Gilman Dr, La Jolla, CA 92093, U.S.A. \\ E-mail: jsong@physics.ucsd.edu
}

ABStRaCt: We study superconformal indices of $4 \mathrm{~d} \mathcal{N}=2$ class $\mathcal{S}$ theories with certain irregular punctures called type $I_{k, N}$. This class of theories include generalized ArgyresDouglas theories of type $\left(A_{k-1}, A_{N-1}\right)$ and more. We conjecture the superconformal indices in certain simplified limits based on the TQFT structure of the class $\mathcal{S}$ theories by writing an expression for the wave function corresponding to the puncture $I_{k, N}$. We write the Schur limit of the wave function when $k$ and $N$ are coprime. When $k=2$, we also conjecture a closed-form expression for the Hall-Littlewood index and the Macdonald index for odd $N$. From the index, we argue that certain short-multiplet which can appear in the OPE of the stress-energy tensor is absent in the $\left(A_{1}, A_{2 n}\right)$ theory. We also discuss the mixed Schur indices for the $\mathcal{N}=1$ class $\mathcal{S}$ theories with irregular punctures.

KEYWORDS: Supersymmetric gauge theory, Supersymmetry and Duality, Topological Field Theories

ARXIV EPrint: 1509.06730 


\section{Contents}

1 Introduction 1

2 Schur index 3

2.1 Wave function for the puncture of type $I_{k, N}$

$\begin{array}{lll}2.2 & \text { Examples } & 7\end{array}$

$\begin{array}{ll}\text { 2.2.1 Lagrangian theories } & 7\end{array}$

2.2.2 Argyres-Douglas theories 8

3 Hall-Littlewood index 10

$\begin{array}{lll}3.1 & \text { Index from the 3d mirror } & 10\end{array}$

$\begin{array}{ll}3.2 \text { Wave function for } I_{2, N} & 12\end{array}$

4 Macdonald index 14

4.1 Wave function for the puncture of type $I_{2, n} \quad 14$

$\begin{array}{lll}4.2 \text { Examples } & 15\end{array}$

$\begin{array}{lll}\text { 4.2.1 Consistency checks } & 16\end{array}$

4.2.2 Conjecture for the Macdonald indices of Argyres-Douglas theories 16

$5 \mathcal{N}=1$ class $\mathcal{S}$ theories $\quad 18$

$\begin{array}{lll}5.1 & \text { Mixed Schur index } & 19\end{array}$

$\begin{array}{lll}5.2 & \mathrm{SU}(2) \mathrm{SYM} \text { with } N_{f}=0,1,2,3 & 20\end{array}$

\section{Introduction}

Four-dimensional $\mathcal{N}=2$ supersymmetric field theories in class $\mathcal{S}$ refers to the ones that can be realized by wrapping $6 \mathrm{~d} \mathcal{N}=(2,0)$ theory on a Riemann surface $\mathcal{C}[1,2]$. This description enables us to understand dynamics of the $4 \mathrm{~d}$ theory in terms of geometry of the Riemann surface. One of the most interesting connection is between the superconformal index $[3,4]$ of the $4 \mathrm{~d}$ theory and the $2 \mathrm{~d}$ topological field theory [5-9]. It says that the superconformal index of a given theory labelled by $\mathcal{C}$ (called the UV curve) is given by a correlation function of the $2 \mathrm{~d}$ topological field theory on $\mathcal{C}$, which is a deformed version of Yang-Mills theory. Especially, in the Schur limit of the index, the TQFT is identified as the $q$-deformed Yang-Mills theory [10]. It has been shown via localization of 5d Maximal SYM on $S^{3}$, that the $2 \mathrm{~d}$ theory is indeed given by the $q$-deformed Yang-Mills theory [11-13]. This relation is also extended to the Lens space index [14-16], to the outer-automorphsim twisted index [17], and to other gauge groups [18-22].

A class $\mathcal{S}$ theory is not just labelled by the UV curve but also its local data on the punctures. There are regular and irregular punctures depending on the boundary condition 
we impose. The regular punctures are labelled by an $\mathrm{SU}(2)$ embedding into $\Gamma$ that labels the $6 \mathrm{~d} \mathcal{N}=(2,0)$ theory. The irregular punctures require more elaborate classifications, and generally lead to non-conformal theories. But when the UV curve is a sphere, we can get a SCFT with one irregular puncture and also with or without one regular puncture $[23,24]$. Theories realized in this way includes Argyres-Douglas theory $[25,26]$ and its generalizations.

The (generalized) Argyres-Douglas theories are inherently strongly-coupled and have no weak-coupling limit. Therefore there has been no direct way of computing the superconformal indices or $S^{1} \times S^{3}$ partition functions. Recently, a progress is made in [27], where they obtained an ansatz for the TQFT description of the Schur index for some of the Argyres-Douglas type theories. They were able to verify their result against S-duality [28] and also by studying dimensional reduction to $3 \mathrm{~d}$ [29]. Their result has been recently extended to the Macdonald index [30]. The result of [27] agrees with the general prediction made in [31], and studied further in [32,33], that for any $\mathcal{N}=2 d=4$ SCFT, there is a protected sector with infinite dimensional chiral algebra acting on it. This implies that the Schur indices have to be given by the vacuum character of the corresponding chiral algebra.

Another progress is made in [34]. They observed that the trace of (inverse) monodromy operator appears in the BPS degeneracy counting [35] agrees with the Schur index (of a non-conformal theory). From this observation, they were able to predict the Schur indices of various generalized Argyres-Douglas theories and conjectured that they are given by the vacuum character of certain non-unitary $W$-minimal models. This agrees with the result of [31], once applied to the Argyres-Douglas theories with no flavor symmetry, yield chiral algebra given by Virasoro/W-algebra with central charges of the minimal model series.

In this paper, we propose the Schur, Hall-Littlewood (HL) and Macdonald limit of the superconformal index for a class $\mathcal{S}$ theory containing certain irregular puncture called (a subset of) type $I_{k, N}$. We conjecture the wave function for irregular puncture in these limits, which enables us to use the TQFT description to compute the indices of AD theories. Our strategy is very similar to $[27,30]$, but we are mainly interested in the theories with no flavor symmetry. It turns out the corresponding wave functions are much simpler than the ones with flavor symmetries. We first start with the irregular punctures that appear in the Lagrangian theories and derive their wave function as an integral transformation of regular punctures. From here, we extrapolate the expression to find a well-behaved wave function corresponding to other irregular punctures. For the Schur index, we are able to find the wave function for arbitrary coprime $k, N$. For the HL index and Macdonald index, we find the wave functions for the $k=2$ cases only.

Curiously, we find that the indices for non-conformal theories can also be written in terms of the TQFT. This is the index of the theory at the UV fixed point (zero coupling) with Gauss law constraint. Even though the theory is non-conformal for non-zero gauge coupling, the UV index is nevertheless well-defined. The TQFT description for the "superconformal index" of a non-conformal $\mathcal{N}=2$ theory enables us to write the index for the conformal $4 \mathrm{~d} \mathcal{N}=1$ class $\mathcal{S}$ theory [36-39].

The outline of this paper is as follows. In section 2, we study Schur index for the theories with $I_{k, N}$ punctures. We are able to give a expression when $k$ and $N$ are coprime, 
and it agrees with other proposals. In section 3, we study Hall-Littlewood index with $I_{2, N}$ punctures. We obtain the wave function for $I_{2, N}$ and compare with the direct computation of the index from the $3 \mathrm{~d}$ mirror theory. In section 4 , we conjecture a closed-form formula for $I_{2, N}$ with $N$ odd and perform a number of consistency checks. In section 5 , we compute mixed Schur limit of the index for $\mathcal{N}=1$ class $\mathcal{S}$ theories using the result of 2 .

\section{Schur index}

Superconformal index of an $\mathcal{N}=2 d=4$ SCFT is defined as

$$
I\left(x_{i} ; p, q, t\right)=\operatorname{Tr}(-1)^{F} p^{j_{2}+j_{1}-r} q^{j_{2}-j_{1}-r} t^{R+r} \prod_{i} x_{i}^{F_{i}},
$$

where $j_{1}, j_{2}$ are the Cartans of the Lorentz group $\mathrm{SU}(2)_{1} \times \mathrm{SU}(2)_{2}$ and $R, r$ are the generators of the $\mathrm{SU}(2)_{R}$ and $\mathrm{U}(1)_{r}$ symmetry respectively. $F_{i}$ are the generators of the flavor symmetries. The trace is taken over the $\frac{1}{8}$-BPS states that are annihilated by a supercharge $Q$. The index can be simplified by taking certain limits [7]. When $p \rightarrow 0$, it is called the Macdonald index and gets contributions from $\frac{1}{4}$-BPS states. The Macdonald index can be further simplified to the Schur limit upon taking $q=t$ limit. The other limit is to take $q \rightarrow 0$, and this is called the Hall-Littlewood (HL) index.

For any class $\mathcal{S}$ theories coming from $6 \mathrm{~d}(2,0)$ theory of type $\Gamma$ wrapped on a Riemann surface $\mathcal{C}_{g, n}$, the superconformal index can be written in terms of a correlation function of a topological field theory

$$
I\left(\boldsymbol{a}_{i} ; p, q, t\right)=\sum_{\boldsymbol{\lambda}} C_{\boldsymbol{\lambda}}^{2 g-2+n} \prod_{i=1}^{n} \psi_{\boldsymbol{\lambda}}^{(i)}\left(\boldsymbol{a}_{i} ; p, q, t\right),
$$

where the sum is over all irreducible representations of $\Gamma$ and $g$ and $n$ is the genus and the number of punctures respectively. The function $C_{\boldsymbol{\lambda}}(p, q, t)$ is sometimes called the structure constant of the theory, and $\psi_{\boldsymbol{\lambda}}(\boldsymbol{a} ; p, q, t)$ is called the wave function we assign to each puncture.

The Schur index is obtained by specialization $p=0, q=t$. In this limit, the structure constant is fixed to be

$$
C_{\boldsymbol{\lambda}}^{-1}=\psi_{\boldsymbol{\lambda}}^{\varnothing}\left(\boldsymbol{a}=q^{\boldsymbol{\rho}} ; q\right)=\frac{\chi_{\boldsymbol{\lambda}}\left(q^{\boldsymbol{\rho}}\right)}{\prod_{i=1}^{r}\left(q^{d_{i}} ; q\right)},
$$

where $\boldsymbol{\rho}$ is the Weyl vector of $\Gamma=A D E$ and $d_{i}$ are the degrees of the Casmirs. We use the short-hand notation $\boldsymbol{z}^{\boldsymbol{a}} \equiv \prod_{i} z_{i}^{a_{i}}$ and $q^{\boldsymbol{\rho}} \equiv \prod_{i} q^{\rho_{i}}$. Here the $q$-Pochhammer symbol is defined as $(z ; q) \equiv \prod_{i=0}^{\infty}\left(1-z q^{i}\right)$. For the case of a full regular puncture, the corresponding wave function is given by

$$
\psi_{\boldsymbol{\lambda}}(\boldsymbol{z} ; q)=\operatorname{PE}\left(\frac{q}{1-q} \chi_{\mathrm{adj}}(\boldsymbol{z})\right) \chi_{\boldsymbol{\lambda}}(\boldsymbol{z})=\frac{1}{(q ; q)^{r} \prod_{\alpha \in \Delta}\left(q \boldsymbol{z}^{\alpha} ; q\right)} \chi_{\boldsymbol{\lambda}}(\boldsymbol{z}),
$$

where $\Delta$ is the set of all $\operatorname{roots}$ of $\Gamma$ and $r=\operatorname{rank}(\Gamma)$. The Plethystic exponent is defined as

$$
\mathrm{PE}[n z]=\frac{1}{(1-z)^{n}},
$$


where $n$ is an integer and $z$ is some fugacity. The wave functions are orthonormal under the vector multiplet measure

$$
\oint[d \boldsymbol{z}] I_{\mathrm{vec}}(\boldsymbol{z}) \psi_{\boldsymbol{\lambda}}(\boldsymbol{z} ; q) \psi_{\boldsymbol{\mu}}(\boldsymbol{z} ; q)=\delta_{\boldsymbol{\lambda}, \boldsymbol{\mu}},
$$

where $[d \boldsymbol{z}]=\prod_{i=1}^{N-1} \frac{d z_{i}}{2 \pi i z_{i}} \Delta(\boldsymbol{z})$ and $\Delta(\boldsymbol{z})=\prod_{\alpha \in \Delta}\left(1-z^{\alpha}\right)$ is the Haar measure. The vector multiplet index is given by

$$
I_{\mathrm{vec}}(\boldsymbol{z})=\operatorname{PE}\left(\frac{-2 q}{1-q} \chi_{\mathrm{adj}}(\boldsymbol{z})\right)=(q ; q)^{2 r} \prod_{\alpha \in \Delta}\left(q \boldsymbol{z}^{\alpha} ; q\right)^{2} .
$$

In addition to the regular punctures, we also have irregular punctures that has higher order singularities. Let us specialize to $\Gamma=A_{k-1}$ and denote the local singularity around the puncture at $z=\infty$ is given as

$$
x^{k}=z^{N}(d z)^{k},
$$

or equivalently around $z^{\prime}=0$ via $z^{\prime}=1 / z$ as

$$
x^{k}=\frac{1}{z^{N+2 k}}\left(d z^{\prime}\right)^{k},
$$

to be $I_{k, N}$, following the notation of $[23,40]$. One can further deform the singularity by adding less singular terms. They serve as deformation parameters of the theory. The regular punctures correspond to $N=-k$. For the case of $k=2$ and $N=2 n$ even, the wave function is written by [27] as

$$
\psi_{\lambda}^{I_{2,2 n}}(a, q)=\frac{q^{(n+1) \frac{\lambda}{2}\left(\frac{\lambda}{2}+1\right)}}{(q ; q)} \operatorname{Tr}_{R_{\lambda}}\left(a^{2 j_{3}} q^{-(n+1) j_{3}^{2}}\right),
$$

where $R_{\lambda}$ is the spin- $\frac{\lambda}{2}$ representation of $\mathrm{SU}(2)$, and $j_{3}=-\frac{\lambda}{2},-\frac{\lambda}{2}+1, \cdots, \frac{\lambda}{2}$.

Our goal in this section is to find a wave function for the punctures of type $I_{k, N}$. For example, we conjecture the wave function for the $I_{2,2 n-1}$ puncture to be

$$
\psi_{\lambda}^{I_{2,2 n-1}}(q)= \begin{cases}(-1)^{\frac{\lambda}{2}} q^{\frac{\lambda}{2}\left(\frac{\lambda}{2}+1\right)\left(n+\frac{1}{2}\right)} & \lambda \text { even, } \\ 0 & \text { otherwise }\end{cases}
$$

as we will provide evidences in this section.

\subsection{Wave function for the puncture of type $I_{k, N}$}

Puncture of type $\boldsymbol{I}_{\boldsymbol{N},-\boldsymbol{N}+\mathbf{1}}$. Let us consider $\mathrm{SU}(N)$ theory with $N$ flavors. This theory can be realized by a 3 -punctured sphere with one maximal, one minimal and one irregular of type $I_{N,-N+1}$. If we write the index of this theory in terms of a TQFT, we get

$$
I(\boldsymbol{a}, x)=\sum_{\boldsymbol{\lambda}} C_{\boldsymbol{\lambda}} \psi_{\boldsymbol{\lambda}}(\boldsymbol{a}) \psi_{\boldsymbol{\lambda}}^{\star}(x) \psi_{\boldsymbol{\lambda}}^{I_{N,-N+1}},
$$


where $\psi_{\boldsymbol{\lambda}}^{\star}(x)$ and $\psi_{\boldsymbol{\lambda}}^{I_{N,-N+1}}$ denote the wave function corresponding to the minimal and $I_{N,-N+1}$ puncture respectively. Since it is the same as gauging one of the flavors of bifundamental hypermutliplets, it can be also written as

$$
I(\boldsymbol{a}, x)=\oint[d \boldsymbol{z}] I_{\mathrm{vec}}(\boldsymbol{z}) \sum_{\boldsymbol{\lambda}} C_{\boldsymbol{\lambda}} \psi_{\boldsymbol{\lambda}}(\boldsymbol{a}) \psi_{\boldsymbol{\lambda}}^{\star}(x) \psi_{\boldsymbol{\lambda}}(\boldsymbol{z}) .
$$

This means that we can simply write the wave function corresponding to the irregular puncture $I_{N,-N+1}$ as $^{1}$

$$
\psi_{\boldsymbol{\lambda}}^{I_{N,-N+1}}(q)=\oint[d \boldsymbol{z}] I_{\mathrm{vec}}(\boldsymbol{z}) \psi_{\boldsymbol{\lambda}}(\boldsymbol{z})=\oint[d \boldsymbol{z}] \mathrm{PE}\left[-\frac{q}{1-q} \chi_{\mathrm{adj}}(\boldsymbol{z})\right] \chi_{\boldsymbol{\lambda}}(\boldsymbol{z})
$$

This integral can be written in a more illuminating form by using the Jacobi triple product identity

$$
(q ; q)\left(y^{-1} ; q\right)(q y ; q)=\sum_{m \in \mathbb{Z}}(-1)^{m} q^{\frac{1}{2} m(m+1)} y^{m} .
$$

We get

$$
\begin{aligned}
\psi_{\boldsymbol{\lambda}}^{I_{N,-N+1}}(q)= & \frac{1}{|\mathcal{W}|(q ; q)^{\frac{1}{2}(N-1)(N-2)}} \\
& \times \sum_{n_{\alpha} \in \mathbb{Z}}(-1)^{\sum_{\alpha} n_{\alpha}} q^{\sum_{\alpha} \frac{1}{2} n_{\alpha}\left(n_{\alpha}+1\right)} \oint d \boldsymbol{z} \prod_{\alpha \in \Delta^{+}}\left(1-\boldsymbol{z}^{\alpha}\right) \boldsymbol{z}^{\sum_{\alpha} n_{\alpha} \alpha} \chi_{\boldsymbol{\lambda}}(\boldsymbol{z}),
\end{aligned}
$$

where $\Delta_{+}$is the set of positive roots of $\mathrm{SU}(N)$ and $\mathcal{W}$ is the Weyl group. Here the index $\alpha$ runs from $\alpha=1, \cdots\left|\Delta_{+}\right|$and the integral measure is given by $d \boldsymbol{z}=\prod_{i=1}^{N-1} \frac{d z_{i}}{2 \pi i z_{i}}$. The last integral can be rewritten upon applying the Weyl character formula as

$$
\sum_{w \in \mathcal{W}} \epsilon(w) \oint d \boldsymbol{z} z^{-\rho+w(\boldsymbol{\lambda}+\rho)-\sum_{\alpha} n_{\alpha} \alpha}=\sum_{w \in \mathcal{W}} \epsilon(w) \delta_{w \cdot \boldsymbol{\lambda}=n_{\alpha} \boldsymbol{\alpha}}^{(N-1)},
$$

where $\rho=\frac{1}{2} \sum_{\alpha \in \Delta_{+}} \alpha$ is the Weyl vector, $\epsilon(w)$ is the signature of $w$ which is the same as the determinant of $w$ and $w \cdot \boldsymbol{\lambda} \equiv w(\boldsymbol{\lambda}+\rho)-\rho$ is the shifted Weyl reflection.

For the case of $N=2$, it is simple to evaluate the above integral. We get

$$
\begin{aligned}
\psi_{\lambda}^{I_{2,-1}}(q) & =\frac{1}{2} \sum_{n \in \mathbb{Z}}(-1)^{n} q^{\frac{1}{2} n(n+1)} \oint \frac{d z}{2 \pi i z}\left(z^{\lambda-2 n}-z^{-\lambda-2 n-2}\right) \\
& = \begin{cases}(-1)^{\frac{\lambda}{2}} q^{\frac{1}{2} \frac{\lambda}{2}\left(\frac{\lambda}{2}+1\right)} & \lambda \text { even }, \\
0 & \lambda \text { odd } .\end{cases}
\end{aligned}
$$

For $N=3$, we find the expression as below:

$$
\psi_{\left(\lambda_{1}, \lambda_{2}\right)}^{I_{3,-2}}(q)= \begin{cases}q^{k(k+1)+\ell(\ell+1)+k \ell} & \text { if } \lambda_{1}=3 k, \lambda_{2}=3 \ell \\ -q^{k^{2}+\ell^{2}-1+(k-1)(\ell-1)} & \text { if } \lambda_{1}=3 k-2, \lambda_{2}=3 \ell-2, \\ 0 & \text { otherwise, }\end{cases}
$$

\footnotetext{
${ }^{1}$ The author would like to thank Yuji Tachikawa for the discussions lead to this observation.
} 
where $k, \ell \in \mathbb{Z}_{\geq 0}$. We do not have an analytic proof of the formula, but we have checked this expression up to $\left(\lambda_{1}, \lambda_{2}\right)=(12,12)$.

This wave function is an analog of Gaiotto-Whittaker vector [41-43] in the AGT correspondence [44, 45], which realize pure YM theory when we have two punctures of this type. In our case, we expect the two point function of Gaiotto-Whittaker state of the $q$-deformed Yang-Mills gives us the 'Schur index' of the pure YM theory ${ }^{2}$

$$
I_{\mathrm{YM}}(q)=\sum_{\boldsymbol{\lambda}} \psi_{\boldsymbol{\lambda}}^{I_{N,-N+1}}(q) \psi_{\boldsymbol{\lambda}}^{I_{N,-N+1}}(q) .
$$

We will prove this relation for the case of $\mathrm{SU}(2)$ in section 2.2.1.

Type $\boldsymbol{I}_{k, N}$ with $(k, N)=1$. Now, we conjecture that the wave function for the irregular puncture of type $I_{k, N}$ when $k$ and $N$ are relative primes, is simply given by rescaling the $q$ parameter of the wave function for the Gaiotto-Whittaker state (2.14). More precisely, we find

$$
\psi_{\boldsymbol{\lambda}}^{I_{k, N}}(q)=\psi_{\boldsymbol{\lambda}}^{I_{k, 1-k}}\left(q^{N+k}\right) .
$$

This can be thought of as an analog of the coherent state considered by [46-48] in the context of AGT correspondence. We give a number of evidences for (2.21) in later sections. It would be desirable to give a direct proof of this proposal.

Puncture of type $\boldsymbol{I}_{\boldsymbol{k}, \boldsymbol{k n}}$. We can also consider a irregular puncture of type $I_{k, N}$ with $N$ being a multiple of $k$. In this case we have $k-1$ mass parameters associated to the $\mathrm{U}(1)^{k-1}$ flavor symmetry. As before, let us first consider a Lagrangian example. Consider $\mathrm{SU}(N)$ gauge theory with $2 N-1$ fundamentals. It is realized by a sphere with a maximal, minimal and $I_{N, 0}$ type irregular punctures. Therefore, we write the wave function for the $I_{N, 0}$ puncture as

$$
\begin{aligned}
\psi_{\boldsymbol{\lambda}}^{I_{N, 0}}(\boldsymbol{a} ; q) & =\oint[d \boldsymbol{z}] I_{\mathrm{vec}}(\boldsymbol{z}) I_{\mathrm{hyp}}(\boldsymbol{z}, \boldsymbol{a}) \psi_{\boldsymbol{\lambda}}(\boldsymbol{z}) \\
& =\oint[d \boldsymbol{z}] \mathrm{PE}\left[-\frac{q}{1-q} \chi_{\mathrm{adj}}(\boldsymbol{z})\right] \operatorname{PE}\left[\frac{q^{\frac{1}{2}}}{1-q} \sum_{i=1}^{N} \sum_{m=1}^{N-1}\left(z_{i} a_{m}+z_{i}^{-1} a_{m}^{-1}\right)\right] \chi_{\boldsymbol{\lambda}}(\boldsymbol{z}) \\
& =\oint[d \boldsymbol{z}](q ; q)^{r} \prod_{i \neq j}\left(q z_{i} / z_{j} ; q\right) \prod_{i=1}^{N} \prod_{m=1}^{N-1} \frac{1}{\left(q^{\frac{1}{2}}\left(z_{i} a_{m}\right)^{ \pm} ; q\right)} \chi_{\boldsymbol{\lambda}}(\boldsymbol{z}),
\end{aligned}
$$

where we impose $\prod_{i} z_{i}=1$ and $\prod_{m} a_{m}=1$. Here \pm means taking product of each sign.

For the case of $N=2$, the wave function is given by [27] as

$$
\psi_{\lambda}^{I_{2,2 n}}(a, q)=\frac{q^{(n+1) \frac{\lambda}{2}\left(\frac{\lambda}{2}+1\right)}}{(q ; q)} \operatorname{Tr}_{R_{\lambda}}\left(a^{2 j_{3}} q^{-(n+1) j_{3}^{2}}\right),
$$

where the trace is over the spin- $\lambda / 2$ representation $R_{\lambda}$. We find that the wave function for the irregular puncture of type $I_{2,2 n}$ can be written almost as a simple rescaling of the $q$ as

$$
\psi_{\lambda}^{I_{2,2 n}}(a ; q)=\frac{\left(q^{n+1} ; q^{n+1}\right)}{(q ; q)} \psi_{\lambda}^{I_{2,0}}\left(a ; q^{n+1}\right) .
$$

\footnotetext{
${ }^{2}$ For a non-conformal theory, the superconformal index really means that of the free UV fixed point with the Gauss-law constraint.
} 
We have checked this expression indeed gives us the same wave function as (2.23) found in [27] to high orders in $q$.

We were not able to find a prescription for $N \geq 3$. It may be possible to find a correct prescription by using isomorphism of $\left(A_{2}, A_{2}\right)=\left(A_{1}, D_{4}\right)$, which has the chiral algebra $\widehat{s u}(3)_{-\frac{3}{2}}$.

\subsection{Examples}

\subsubsection{Lagrangian theories}

SU(2) SYM. Pure SU(2) YM theory can be realized on a sphere with 2 irregular punctures with $x^{2} \propto\left(d z^{\prime}\right)^{2} / z^{\prime 3}$, which means $I_{2,-1}$. From the TQFT, we get

$$
I_{\mathrm{TQFT}(-1,-1)}(q)=\sum_{\lambda} \psi_{\lambda}^{I_{2,-1}} \psi_{\lambda}^{I_{2,-1}}=\sum_{j \in \mathbb{Z}_{\geq 0}} q^{j(j+1)} .
$$

It agrees with the integral expression obtained from blindly applying the index formula for the vector multiplets and then integrating over the gauge group

$$
I_{\mathrm{SYM}}(q)=(q ; q)^{2} \oint \frac{d z}{2 \pi i z} \Delta(z)\left(q z^{ \pm 2} ; q\right)^{2}=\sum_{m \in \mathbb{Z}_{\geq 0}} q^{m(m+1)},
$$

where $\Delta(z)=\frac{1}{2}\left(1-z^{ \pm 2}\right)$ is the Haar measure of $\mathrm{SU}(2)$. This integral can be easily evaluated by using the Jacobi triple identity (2.15). It can be considered as the index at the UV fixed point with Gauss law constraint.

SU(2) with $\boldsymbol{N}_{\boldsymbol{f}}=\mathbf{1}$. The $\mathrm{SU}(2)$ gauge theory with 1 flavor can be realized by a sphere with 2 irregular punctures $I_{2,-1}$ and $I_{2,0}$. From the TQFT, we get

$$
\begin{aligned}
I_{\mathrm{TQFT}(-1,0)}(q, a)= & \sum_{\lambda} \psi_{\lambda}^{I_{2,-1}} \psi_{\lambda}^{I_{2,0}}(a) \\
= & 1+q+\left(-a^{2}-\frac{1}{a^{2}}+2\right) q^{2}+\left(-a^{2}-\frac{1}{a^{2}}+2\right) q^{3} \\
& +\left(-2 a^{2}-\frac{2}{a^{2}}+4\right) q^{4}+\left(a^{4}+\frac{1}{a^{4}}-3 a^{2}-\frac{3}{a^{2}}+5\right) q^{5}+O\left(q^{6}\right),
\end{aligned}
$$

which agrees with the one computed from the integral

$$
I_{N_{f}=1}=(q ; q)^{2} \oint \frac{d z}{2 \pi i z} \Delta(z) \frac{\left(q z^{ \pm 2} ; q\right)^{2}}{\left(q^{\frac{1}{2}} z^{ \pm} a^{ \pm} ; q\right)} .
$$

SU(2) with $\boldsymbol{N}_{\boldsymbol{f}}=\mathbf{2}$. The $\mathrm{SU}(2)$ gauge theory with 2 flavors can be realized by a sphere with 2 irregular punctures $I_{2,0}$. This gives us

$$
\begin{aligned}
I_{\mathrm{TQFT}(0,0)}= & \sum_{\lambda} \psi_{\lambda}^{I_{2,0}}(a) \psi_{\lambda}^{I_{2,0}(b)} \\
= & 1+q\left(\chi_{[1,0]}^{\mathrm{SO}(4)}+\chi_{[0,1]}^{\mathrm{SO}(4)}+2 \chi_{[0,0]}^{\mathrm{SO}(4)}\right)+q^{2}\left(\chi_{[2,0]}^{\mathrm{SO}(4)}+\chi_{[0,2]}^{\mathrm{SO}(4)}+2 \chi_{[1,0]}^{\mathrm{SO}(4)}+2 \chi_{[0,1]}^{\mathrm{SO}(4)}+3 \chi_{[0,0]}^{\mathrm{SO}(4)}\right) \\
& +q^{3}\left(\chi_{[3,0]}^{\mathrm{SO}(4)}+\chi_{[0,3]}^{\mathrm{SO}(4)}+2 \chi_{[2,0]}^{\mathrm{SO}(4)}+2 \chi_{[0,2]}^{\mathrm{SO}(4)}+4 \chi_{[1,0]}^{\mathrm{SO}(4)}+4 \chi_{[0,1]}^{\mathrm{SO}(4)}+6 \chi_{[0,0]}^{\mathrm{SO}(4)}\right)+O\left(q^{4}\right),
\end{aligned}
$$


which agrees with the computation from the integral formula. Here we used the Dynkin label to denote the characters.

We can also realize the same theory via 3-punctured sphere with 2 regular punctures and one $I_{2,-1}$ puncture. This gives

$$
I_{\mathrm{TQFT}(R, R,-1)}(x, y ; q)=\sum_{\lambda} C_{\lambda} \psi_{\lambda}(x) \psi_{\lambda}(y) \psi_{\lambda}^{I_{2,-1}}
$$

which gives the same answer upon matching the fugacities via $a \rightarrow \sqrt{x y}$ and $b \rightarrow \sqrt{x / y}$.

$\mathbf{S U}(2)$ with $\boldsymbol{N}_{\boldsymbol{f}}=\mathbf{3}$. The $\mathrm{SU}(2)$ gauge theory with 3 flavors can be realized by a sphere with 2 regular punctures and 1 irregular puncture $I_{2,0}$. This gives us the index to be

$$
\begin{aligned}
I_{\mathrm{TQFT}(R, R, 0)}= & \sum_{\lambda} C_{\lambda} \psi_{\lambda}(a) \psi_{\lambda}(b) \psi_{\lambda}^{I_{2,0}}(c) \\
= & 1+q \chi_{[0,1,1]}^{\mathrm{SO}(6)}+q^{2}\left(\chi_{[0,2,2]}^{\mathrm{SO}(6)}+\chi_{[0,1,1]}^{\mathrm{SO}(6)}+\chi_{[0,0,0]}^{\mathrm{SO}(6)}\right) \\
& +q^{3}\left(\chi_{[0,3,3]}^{\mathrm{SO}(6)}+\chi_{[0,2,2]}^{\mathrm{SO}(6)}+\chi_{[1,2,0]}^{\mathrm{SO}(6)}+\chi_{[1,0,2]}^{\mathrm{SO}(6)}+\chi_{[0,1,1]}^{\mathrm{SO}(6)}+\chi_{[0,0,0]}^{\mathrm{SO}(6)}\right)+O\left(q^{4}\right) .
\end{aligned}
$$

This agrees with the integral formula

$$
I_{N_{f}=3}\left(a_{i} ; q\right)=(q ; q)^{2} \oint \frac{d z}{2 \pi i z} \Delta(z) \frac{\left(q z^{ \pm 2} ; q\right)^{2}}{\prod_{i=1}^{3}\left(q^{\frac{1}{2}} z^{ \pm} a_{i}^{ \pm} ; q\right)},
$$

upon identifying $a_{1}=b c, a_{2}=b / c, a_{3}=a$.

SU(3) SYM. The Schur index of the SU(3) pure YM can be written as

$$
\begin{aligned}
I_{\mathrm{SU}(3)} & =\frac{1}{3 !}(q ; q)^{4} \oint \frac{d z_{1}}{2 \pi i z_{1}} \frac{d z_{2}}{2 \pi i z_{2}} \prod_{\alpha \in \Delta_{+}}\left(1-z^{ \pm \alpha}\right)\left(q z^{ \pm \alpha} ; q\right)^{2} \\
& =\frac{1}{6(q ; q)^{2}} \sum_{n_{1,2,3}, m_{1,2,3} \in \mathbb{Z}}(-1)^{n_{1}+m_{1}} q^{\frac{1}{2} \sum_{i}\left(n_{i}\left(n_{i}+1\right)+m_{i}\left(m_{i}+1\right)\right)} \delta_{n_{1}+n_{2}, m_{1}+m_{2}} \delta_{n_{1}+n_{3}, m_{1}+m_{3}} .
\end{aligned}
$$

We verified this to be the same as the one given by (2.20) with $N=3$ to high orders in $q$. The first few terms are

$$
I_{\mathrm{SU}(3)}=1+q^{2}+2 q^{4}+2 q^{8}+q^{10}+2 q^{12}+q^{16}+2 q^{18}+2 q^{20}+O\left(q^{24}\right) .
$$

\subsubsection{Argyres-Douglas theories}

$\left(\boldsymbol{A}_{1}, \boldsymbol{A}_{N-1}\right)$ theories. The Argyres-Douglas theories of type $\left(A_{1}, A_{N-1}\right)$ can be realized by a sphere with single irregular puncture of type $I_{2, N}[23,40]$. When $N=2 n+1$ is odd, we have

$$
I_{A_{2 n}}(q)=\sum_{\lambda} C_{\lambda}^{-1} \psi_{\lambda}^{I_{2,2 n+1}}=\sum_{j \in \mathbb{Z}_{\geq 0}} \frac{[2 j+1]_{q}}{\left(q^{2} ; q\right)}(-1)^{j} q^{j(j+1)\left(n+\frac{1}{2}\right)},
$$

where

$$
C_{\lambda}^{-1}=\frac{[\operatorname{dim} \lambda]_{q}}{\left(q^{2} ; q\right)}, \quad[n]_{q}=\frac{q^{\frac{n}{2}}-q^{-\frac{n}{2}}}{q^{\frac{1}{2}}-q^{-\frac{1}{2}}}
$$


The vacuum character of the Virasoro minimal model $(r, s)$ is given by

$$
\chi_{0}^{(r, s)}(q)=\frac{q^{-\frac{1}{4} \frac{(r-s)^{2}}{r s}}}{(q ; q)} \sum_{\ell \in \mathbb{Z}}\left(q^{\frac{(2 r s \ell+r-s)^{2}}{4 r s}}-q^{\frac{(2 r s \ell+r+s)^{2}}{4 r s}}\right) .
$$

We have checked this expression agrees with the vacuum character of the Virasoro minimal model $(2,2 n+3)$ to high orders in $q$, which is consistent with the relation given in [31], and also computation based on the conjectural relation from the BPS degeneracy [34]. We also find that when $n=0$ or $N=1$, the index becomes 1 , which agrees with the expectation that there is no massless degrees of freedom and vanishing central charges [40] for the $\left(A_{1}, A_{0}\right)$ theory.

When $N=2 n$ is even, we get [27]

$$
I_{A_{2 n-1}}(x ; q)=\sum_{\lambda} C_{\lambda}^{-1} \psi_{\lambda}^{I_{2,2 n}}(x)=\sum_{\lambda \geq 0} \frac{[\lambda+1]_{q}}{\left(q^{2} ; q\right)} \operatorname{Tr}_{R_{\lambda}}\left(x^{2 j_{3}} q^{(n+1)\left(\frac{\lambda}{2}\left(\frac{\lambda}{2}+1\right)-j_{3}^{2}\right)}\right),
$$

which agrees with the vacuum character of $\widehat{s u}(2)_{-\frac{4}{3}}$ when $n=2$.

$\left(\boldsymbol{A}_{\mathbf{1}}, \boldsymbol{D}_{\boldsymbol{N}+\mathbf{2}}\right)$ theories. The AD theory of type $\left(A_{1}, D_{N+2}\right)$ can be realized by a sphere with one $I_{2, N}$ type irregular puncture and a regular puncture. When $N=2 n-1$, the index can be written as

$$
I_{\left(A_{1}, D_{2 n+1}\right)}=\sum_{\lambda} \psi_{\lambda}^{I_{2,2 n-1}} \psi_{\lambda}(x)=\frac{1}{\left(q x^{ \pm 2,0} ; q\right)} \sum_{m=0}^{\infty}(-1)^{m} q^{\frac{m(m+1)}{2}(2 n+1)} \chi_{R_{2 m}}(x) .
$$

This expression exactly agrees with the vacuum character of the affine Lie algebra $\widehat{s u}(2)_{-\frac{4 n}{2 n+1}}$. When $N=1$, we find the index of $\left(A_{1}, A_{3}\right)$ agrees with that of $\left(A_{1}, D_{3}\right)$

$$
I_{\left(A_{1}, A_{3}\right)}=\sum_{\lambda} C_{\lambda}^{-1} \psi_{\lambda}^{I_{2,4}}(x)=\sum_{\lambda} \psi_{\lambda}^{I_{2,1}} \psi_{\lambda}(a)=I_{\left(A_{1}, D_{3}\right)},
$$

up on identifying $x=a^{2}$.

When $N=2 n$, the index can be written as

$$
I_{\left(A_{1}, D_{2 n+2}\right)}=\sum_{\lambda} \psi_{\lambda}^{I_{2,2 n}}(x) \psi_{\lambda}(y) .
$$

When $n=1$, this agrees with the vacuum character of $\widehat{s u}(3)_{-\frac{3}{2}}[27]$.

$\left(\boldsymbol{A}_{\boldsymbol{k}-1}, \boldsymbol{A}_{N-1}\right)$ theories with $(\boldsymbol{k}, \boldsymbol{N})=1$. When $k$ and $N$ are coprime, we conjecture that the Schur indices for the Argyres-Douglas theories of type $\left(A_{k-1}, A_{N-1}\right)$ as

$$
I_{\left(A_{k-1}, A_{N-1}\right)}(q)=\frac{1}{\prod_{i=2}^{k}\left(q^{i} ; q\right)} \sum_{\boldsymbol{\lambda}} \chi_{\boldsymbol{\lambda}}\left(q^{\rho}\right) \psi_{\boldsymbol{\lambda}}^{I_{k, N}}(q) .
$$

It was conjectured by [34] that for the coprime $k, N$, the Schur index of $\left(A_{k-1}, A_{N-1}\right)$ theory is given by the vacuum character of the $(k, k+N) W_{k}$-minimal model, which is given by [49]

$$
\chi_{0}^{W(k, k+N)}(q)=\left(\frac{\left(q^{k+N} ; q^{k+N}\right)}{(q ; q)}\right)^{k-1} \prod_{a=1}^{k-1}\left(q^{N+a} ; q^{k+N}\right)^{a}\left(q^{a} ; q^{k+N}\right)^{k-a} .
$$

We checked $I_{\left(A_{k-1}, A_{N-1}\right)}(q)=\chi_{0}^{W(k, k+N)}(q)$ to high orders in $q$ for a number of cases. 


\section{Hall-Littlewood index}

\subsection{Index from the 3d mirror}

For Argyres-Douglas theories realized in class $\mathcal{S}$ with a non-trivial Higgs branch, ${ }^{3}$ once dimensionally reduced to $3 \mathrm{~d}$, their mirror theories [51] are known [23, 52]. The Higgs branch of the dimensionally reduced $3 \mathrm{~d} \mathcal{N}=4$ theory is the same as the original $4 \mathrm{~d} \mathcal{N}=2$ theory, which is the same as the Coulomb branch of the $3 \mathrm{~d}$ mirror

$$
\mathcal{M}_{\text {Higgs }}^{4 d}=\mathcal{M}_{\text {Higgs }}^{3 d}=\mathcal{M}_{\text {Coulomb }}^{3 d \text { mirror }} .
$$

Since the Hall-Littlewood (HL) index is the same as the Hilbert series of the Higgs branch [7], it should be the same as the Hilbert series of the Coulomb branch [53] of the $3 \mathrm{~d}$ mirror theory. This can be also computed using the $3 \mathrm{~d}$ superconformal index by taking the 'Coulomb limit' as discussed in [54]. Therefore we can write

$$
I_{\mathrm{HL}}^{4 d}(t)=I_{\mathrm{Higgs}}^{3 d}(t)=I_{\text {Coulomb }}^{3 d \text { mirror }}(t) .
$$

In this section, we review the computation of HL indices from the $3 \mathrm{~d}$ mirrors [55].

The Coulomb branch index for the $3 \mathrm{~d} \mathcal{N}=4$ theory is defined as

$$
I^{C}(t)=\operatorname{Tr}(-1)^{F} t^{E-R_{H}}=\operatorname{Tr}(-1)^{F} t^{E+j},
$$

where the trace is over the states with $E=R_{C}$ in addition to $E-R_{H}-E_{C}-j=0$. Here $R_{H}, R_{C}$ are the Cartans of $\mathrm{SO}(4)_{R}=\mathrm{SU}(2)_{R_{H}} \times \mathrm{SU}(2)_{R_{C}}$ and $E$ is the scaling dimension and $j$ is the Cartan of the rotation group $\mathrm{SO}(3)$. The Coulomb branch index contribution for a hypermultiplet is simply

$$
I_{\text {hyp }}^{C}(z, m ; t)=t^{\frac{1}{2}|m|},
$$

which does not depend on any flavor fugacities. Here $m$ is the charge for the background topological U(1) associated to the gauge group.

$\left(\boldsymbol{A}_{1}, \boldsymbol{A}_{2 n-1}\right)$ theories. The $3 \mathrm{~d}$ mirror theory is given by a quiver gauge theory with $k$ $\mathrm{U}(1)$ nodes where all the nodes are connected by $n$ edges. One of the $\mathrm{U}(1)$ node has to be ungauged as usual. See the figure 1. For the U(1) gauge theory with $n$ fundamental hypers, the index is given as

$$
I_{\left(A_{1}, A_{2 n-1}\right)}^{C}(w ; t)=\frac{1}{1-t} \sum_{m \in \mathbb{Z}} w^{m} t^{\frac{n}{2}|m|},
$$

where $w$ is the fugacity for the topological $\mathrm{U}(1)_{J}$ associated to the gauge group. One can sum up the formula to get

$$
I_{n}^{C}(w ; t)=\left(\frac{1-t^{n}}{1-t}\right) \frac{1}{\left(1-t^{\frac{n}{2}} w\right)\left(1-t^{\frac{n}{2}} w^{-1}\right)}=\left(\frac{1-t^{n}}{1-t}\right) \sum_{k=0}^{\infty} \chi_{[k]}^{\mathrm{SU}(2)}(w) t^{k n / 2},
$$

where $\chi_{[k]}$ denotes the characters of $\mathrm{SU}(2)$ with Dynkin label $[k]$. Even though we see the index is written in terms of characters $\mathrm{SU}(2)$, it is wrong to say that the global symmetry is actually enhanced to $\mathrm{SU}(2)$. Note that only when $n=2$, we have the extra conserved current coming from the monopoles [56].

\footnotetext{
${ }^{3}$ Even though the theory in the UV might not have a Higgs branch, AD points can sometimes have a quantum Higgs branch [50].
} 


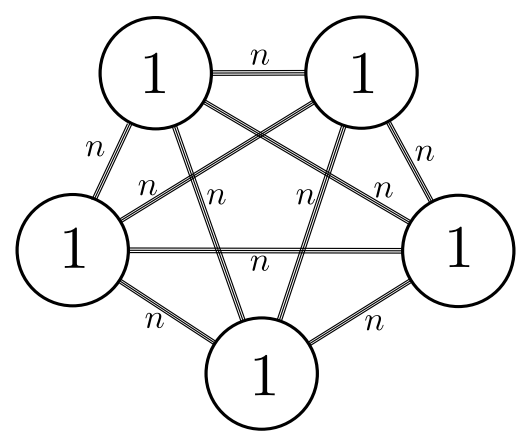

Figure 1. The $3 \mathrm{~d}$ mirror of $\left(A_{k-1}, A_{k n-1}\right)$ theory. $k$ is the number of nodes and $n$ is the number of bifundamentals between a pair of nodes. One of the $\mathrm{U}(1)$ has to be decoupled.

The Coulomb branch of $\mathrm{U}(1)$ gauge theory with $n$ electrons or the Higgs branch of its mirror theory $\hat{A}_{n-1}$ quiver is known to be given by $\mathbb{C}^{2} / \mathbb{Z}_{n}$. One can directly see that the Hilbert series of this space is the same as above.

For $n=2$, the $3 \mathrm{~d}$ theory is well-known $T[\mathrm{SU}(2)]$ theory which is self-mirror. Here the $\mathrm{SU}(2)$ enhancement of topological symmetry is evident from the mirror perspective. There $\mathrm{SU}(2)$ symmetry is nothing but the flavor symmetry of two electrons. In this case, the $4 \mathrm{~d}$ theory is the same as the AD theory found from $\mathrm{SU}(2)$ gauge theory with two flavors.

$\left(\boldsymbol{A}_{2}, \boldsymbol{A}_{3 n-1}\right)$ theories. The $3 \mathrm{~d}$ mirror of this theory is given by $\mathrm{U}(1) \times \mathrm{U}(1)$ gauge theory with $n$ bifundamentals and $n$ electrons for each $\mathrm{U}(1)$. The Coulomb branch index is given by

$$
I_{\left(A_{2}, A_{3 n-1}\right)}^{C}\left(w_{1}, w_{2} ; t\right)=\frac{1}{(1-t)^{2}} \sum_{m_{1}, m_{2} \in \mathbb{Z}} w_{1}^{m_{1}} w_{2}^{m_{2}} t^{\frac{1}{2}\left(\left|m_{1}\right|+\left|m_{2}\right|+\left|m_{1}-m_{2}\right|\right) n},
$$

which can be written in terms of $\mathrm{SU}(3)$ characters as

$$
I_{\left(A_{2}, A_{3 n-1}\right)}^{C}(\boldsymbol{a} ; t)=\left(\frac{1-t^{n}}{1-t}\right)^{2} \sum_{k=0}^{\infty} \chi_{[k, k]}^{\mathrm{SU}(3)}(\boldsymbol{a}) t^{n k}
$$

where $[k, k]$ is the Dynkin label for the $k$-th powers of adjoint representation of $\mathrm{SU}(3)$ and the fugacities are mapped to $w_{1}=a_{1} / a_{2}^{2}, w_{2}=a_{2} / a_{1}^{2}$. From above, we see that the global symmetry is enhanced to $\mathrm{SU}(3)$.

This flavor symmetry can be understood quite easily when $n=1$. In this case, the quiver gauge theory we obtain is nothing but $\hat{A}_{2}$ quiver theory with $\mathrm{U}(1)$ nodes, which is mirror to the $\mathrm{U}(1)$ gauge theory with 3 electrons. As in the previous case, we have $\mathrm{SU}(3)$ flavor symmetry for $n=1$, which gives us extra conserved current from the monopole operators. For $n>1$, even though we get $\mathrm{SU}(3)$ character representations, it does not mean that our theory has extra conserved currents.

Curiously, we observe that the $3 \mathrm{~d}$ mirror of $\left(A_{2}, A_{2}\right)$ theory is simply given by $\hat{A}_{2}$ quiver theory, which is mirror to $\mathrm{U}(1)$ with 3 electrons, which is the $3 \mathrm{~d}$ mirror of $\left(A_{1}, A_{3}\right)$ theory. This is not a surprise, in the sense that generally in class $\mathcal{S}$, there can be many different ways to realize the same $4 \mathrm{~d}$ SCFT. See [57] for a study of such examples among the $\mathrm{AD}$ theories. 


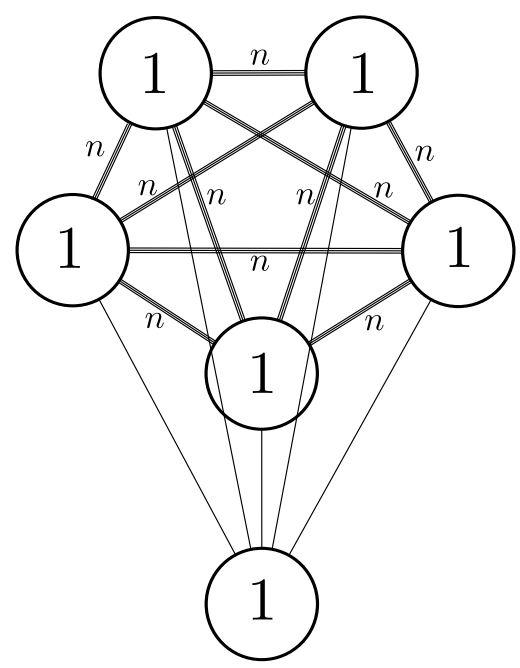

Figure 2. The $3 \mathrm{~d}$ mirror of $\left(A_{k-1}, D_{k n+2}\right)$ theory. The overall $\mathrm{U}(1)$ has to be decoupled as before.

$\left(\boldsymbol{A}_{1}, \boldsymbol{D}_{\mathbf{2 n + 2}}\right)$ theories. This theory is obtained by putting extra U(1) punctures to $\left(A_{1}, A_{2 n-1}\right)$ theory. The $3 \mathrm{~d}$ mirror is obtained by gluing the $3 \mathrm{~d}$ mirror theory corresponding to the minimal puncture to the $3 \mathrm{~d}$ mirror of $\left(A_{k-1}, A_{k n-1}\right)$ theory. See the figure 2 . The Coulomb index for the mirror theory is given as

$$
I_{\left(A_{1}, D_{2 n+2}\right)}^{C}=\frac{1}{(1-t)^{2}} \sum_{m_{1}, m_{2}} w_{1}^{m_{1}} w_{2}^{m_{2}} t^{\frac{1}{2}\left(n\left|m_{1}-m_{2}\right|+\left|m_{1}\right|+\left|m_{2}\right|\right)} .
$$

When $n=1$, the above sum can be written as

$$
I_{\left(A_{1}, D_{4}\right)}^{C}=\sum_{k} \chi_{[k, k]}^{\mathrm{SU}(3)}(\boldsymbol{a}) t^{k},
$$

which is the same as that of $\left(A_{2}, A_{2}\right)$ theory as expected.

\subsection{Wave function for $I_{2, N}$}

$\boldsymbol{N}=\mathbf{2} \boldsymbol{n}$ even. We want to write the index (3.5) using the TQFT as

$$
I_{\left(A_{1}, A_{2 n-1}\right)}(a)=\sum_{\lambda} C_{\lambda}^{-1} \psi_{\lambda}^{I_{2,2 n}}(a)
$$

with

$$
C_{\lambda}=\frac{\prod_{i=1}^{r}\left(1-t^{d_{i}}\right)}{P_{\lambda}^{\mathrm{HL}}\left(t^{\frac{1}{2}}\right)},
$$

where $d_{i}$ are the degrees of the Casmirs of $\Gamma$. For $\Gamma=\mathrm{SU}(N)$, they are given by $d_{i}=$ $2,3, \cdots, N$. Here the function $P_{\lambda}^{\mathrm{HL}}(a)$ is the Hall-Littlewood polynomial labelled by a Dynkin label $\boldsymbol{\lambda}$ in general, which we normalize so that

$$
\left\langle P_{\boldsymbol{\lambda}}^{\mathrm{HL}}(z), P_{\boldsymbol{\mu}}^{\mathrm{HL}}(z)\right\rangle=\int[d z] \Delta(z) \mathrm{PE}\left[t \chi_{\mathrm{adj}}(\boldsymbol{z})\right] P_{\boldsymbol{\lambda}}^{\mathrm{HL}}(z) P_{\boldsymbol{\mu}}^{\mathrm{HL}}(z)=\delta_{\lambda \mu} .
$$


For the case of $\Gamma=\mathrm{SU}(2)$, we get

$$
P_{\lambda}^{\mathrm{HL}}(z)= \begin{cases}\sqrt{1-t^{2}} & \text { if } \lambda=0 \\ \sqrt{1-t}\left(\chi_{\lambda}(z)-t \chi_{\lambda-2}(z)\right) & \text { if } \lambda \neq 0 .\end{cases}
$$

The wave function for the $I_{2,2 n}$ puncture can be read off from the expression (3.5) to be

$$
\psi_{\lambda}^{I_{2,2 n}}(a)= \begin{cases}\sqrt{\frac{1+t}{1-t}} & \text { if } \lambda=0, \\ \left(a^{m}+a^{-m}\right) \frac{t^{\frac{\lambda}{2}}(n+1)}{\sqrt{1-t}} & \text { otherwise. }\end{cases}
$$

Note that there are many different ways decompose the sum (3.5) in the form of (3.11). But we find our particular form given by (3.15) is the right choice to maintain the consistent TQFT description of the index. We find that this is indeed consistent with the accidental isomorphism of the $\mathrm{AD}$ theories.

One can obtain the wave function for the $I_{2,0}$ puncture as in the previous section by integrating the wave function for the regular puncture with the integration kernel given by vector and hypermultiplets

$$
\begin{aligned}
\psi_{\lambda}^{I_{2,0}} & =\oint[d z] I_{\mathrm{vec}}(z) I_{\mathrm{hyp}}(z, a) \psi_{\lambda}(z) \\
& =\oint[d z] \mathrm{PE}\left[t \chi_{\mathrm{adj}}(z)\right] \mathrm{PE}\left[t^{\frac{1}{2}}\left(z+\frac{1}{z}\right)\left(a+\frac{1}{a}\right)\right] P_{\lambda}^{\mathrm{HL}}(z),
\end{aligned}
$$

where the wave function for the regular puncture is given as $\psi_{\lambda}(a)=\mathrm{PE}\left[t \chi_{\mathrm{adj}}(a)\right] P_{\lambda}^{\mathrm{HL}}(a)$. We find it agrees with the expression (3.15) with $n=0$.

From the TQFT structure of the index, the index for the $\left(A_{1}, D_{2 n+2}\right)$ theory can be written as

$$
I_{\left(A_{1}, D_{2 n+2}\right)}=\sum_{\lambda} \psi_{\lambda}^{I_{2,2 n}}(x) \psi_{\lambda}(y) .
$$

We indeed find this expression agrees with the result (3.9) from the $3 \mathrm{~d}$ mirror.

$\boldsymbol{N}=\mathbf{2 n}-\mathbf{1}$ odd. Again, one can obtain the wave function for $I_{2,-1}$ by integrating the regular puncture wave function via vector multiplet measure. We get

$$
\begin{aligned}
\psi_{\lambda}^{I_{2,-1}} & =\oint[d z] I_{\mathrm{vec}}(z) \psi_{\lambda}(z)=\oint[d z] \operatorname{PE}\left[t \chi_{\mathrm{adj}}(z)\right] P_{\lambda}^{\mathrm{HL}}(z) \\
= & \begin{cases}\sqrt{1-t^{2}} & \lambda=0 \\
-t \sqrt{1-t^{2}} & \lambda=2 \\
0 & \text { otherwise }\end{cases}
\end{aligned}
$$

From this, we obtain the HL index for the pure SU(2) YM to be

$$
I_{\mathrm{SYM}}(t)=\sum_{\lambda} \psi_{\lambda}^{I_{2,-1}} \psi_{\lambda}^{I_{2,-1}}=1-t^{3},
$$

which agrees with the direct computation. 
We can obtain the wave function for $I_{2,1}$ from using the isomorphism $\left(A_{1}, A_{3}\right)=$ $\left(A_{1}, D_{3}\right)$. Note that it implies

$$
\sum_{\lambda} C_{\lambda}^{-1} \psi_{\lambda}^{I_{2,4}}(x)=\sum_{\lambda} \psi_{\lambda}^{I_{2,1}} \psi_{\lambda}(z)
$$

where we identify $x=z^{2}$ as we learned from the Schur index. Now, we can use the orthonormality of the regular puncture wave functions. Multiply $\psi_{\rho}(z)$ on both sides and then integrate with the vector multiplet measure to obtain

$$
\psi_{\rho}^{I_{2,1}}=\sum_{\lambda} \oint[d z] I_{\mathrm{vec}}(z) C_{\lambda}^{-1} \psi_{\lambda}^{I_{2,4}}\left(z^{2}\right) \psi_{\rho}(z)=\sqrt{1-t^{2}} \delta_{\rho, 0} .
$$

We conjecture the Hall-Littlewood wave function for $I_{2,2 n-1}$ is the same as that of $I_{2,1}$, so that

$$
\psi_{\lambda}^{I_{2,2 n-1}}=\sqrt{1-t^{2}} \delta_{\lambda, 0},
$$

for $n>0$. This gives the HL index for the $\left(A_{1}, A_{2 n}\right)$ theory to be 1 , which is consistent with the absence of the Higgs branch.

\section{Macdonald index}

In this section, we discuss the Macdonald index, carrying two fugacities $(q, t)$. The Macdonald index reduces to the Schur index when $q=t$ and to the Hall-Littlewood index when $q=0$. We first construct the wave function for the puncture of type $I_{2, n}$ and then use it to write the Macdonald index for the $\left(A_{1}, A_{2 n}\right)$ theory and discuss its implications.

\subsection{Wave function for the puncture of type $I_{2, n}$}

Odd $\boldsymbol{n}$. As in the previous sections, we start with the $I_{2,-1}$ puncture. It can be obtained by a 'integral transformation' of a regular puncture via vector multiplet measure. The wave function for the regular puncture is given by

$$
\psi_{\boldsymbol{\lambda}}(\boldsymbol{z})=\mathrm{PE}\left[\frac{t}{1-q} \chi_{\mathrm{adj}}(\boldsymbol{z})\right] \underline{P}_{\boldsymbol{\lambda}}(\boldsymbol{z} ; q, t)=\frac{1}{(t ; q)^{r}} \prod_{\boldsymbol{\alpha} \in \Delta} \frac{1}{\left(t \boldsymbol{z}^{\boldsymbol{\alpha}} ; q\right)} \underline{P}_{\boldsymbol{\lambda}}(\boldsymbol{z} ; q, t),
$$

where $\underline{P}_{\boldsymbol{\lambda}}(\boldsymbol{z} ; q, t)=N_{\boldsymbol{\lambda}} P_{\boldsymbol{\lambda}}(\boldsymbol{z} ; q, t)$ is the normalized Macdonald polynomial ${ }^{4}$ such that

$$
\left\langle\psi_{\boldsymbol{\lambda}}, \psi_{\boldsymbol{\mu}}\right\rangle=\oint[d \boldsymbol{z}] I_{\mathrm{vec}}(\boldsymbol{z}) \psi_{\boldsymbol{\lambda}}(\boldsymbol{z}) \psi_{\boldsymbol{\mu}}(\boldsymbol{z})=\delta_{\boldsymbol{\lambda} \boldsymbol{\mu}},
$$

where the normalization factor for the $\Gamma=A_{1}$ is given by

$$
N_{\lambda}(q, t)=\left(\frac{(q ; q)\left(t^{2} q^{\lambda} ; q\right)}{(t ; q)\left(q^{\lambda+1} ; q\right)}\left(1-t q^{\lambda}\right)\right)^{\frac{1}{2}} .
$$

\footnotetext{
${ }^{4}$ Our normalization is slightly different from the usual one. We include the 'Cartan piece' $\frac{(q ; q)^{r}}{(t ; q)^{r}}$ to the measure.
} 
From now on we suppress the dependence on $q, t$. When $\Gamma=A_{1}$, we can explicitly write down the polynomial as

$$
\underline{P}_{\lambda}(a)=N_{\lambda} \sum_{i=0}^{\lambda} \frac{(t ; q)_{i}}{(q ; q)} \frac{(t ; q)_{\lambda-i}}{(q ; q)_{\lambda-i}} a^{2 i-\lambda}
$$

where we define the $q$-Pochhammer symbol as $(x ; q)_{n}=\prod_{i=0}^{n-1}\left(1-x q^{i}\right)$.

Now, let us compute the wave function for the Gaiotto-Whittaker state for the $\Gamma=A_{1}$ theory. We evaluate the integral to get

$$
\begin{aligned}
\psi_{\lambda}^{I_{2,-1}}(q, t) & =\oint[d z] I_{\mathrm{vec}}(z) \psi_{\lambda}(z ; q, t)=\oint[d z] \mathrm{PE}\left(\frac{-q-t}{1-q} \chi_{\mathrm{adj}}(z)\right) \operatorname{PE}\left[\frac{t}{1-q} \chi_{\mathrm{adj}}(z)\right] \underline{P}_{\lambda}(z) \\
& =N_{\lambda} \sum_{i=0}^{\lambda} \frac{(t ; q)_{i}(t ; q)_{\lambda-i}}{(q ; q)_{i}(q ; q)_{\lambda-i}} \oint \frac{d z}{2 \pi i z} \frac{\left(1-z^{ \pm 2}\right)}{2}\left(q z^{ \pm 2,0} ; q\right) z^{2 i-\lambda} \\
& = \begin{cases}(-1)^{\frac{\lambda}{2}} t^{\frac{\lambda}{2}} q^{\frac{1}{2} \frac{\lambda}{2}\left(\frac{\lambda}{2}-1\right)} N_{\lambda} \frac{(t ; q)_{\lambda / 2}}{(q ; q)_{\lambda / 2}} & \lambda \text { even, } \\
0 & \lambda \text { odd. }\end{cases}
\end{aligned}
$$

We see that Schur and Hall-Littlewood limits of (4.5) indeed reproduces the corresponding wave functions.

From (4.5), we want to find a similar rescaling of the fugacities $(q, t)$ as done in the Schur and Hall-Littlewood case. Now, we conjecture that the wave function for $I_{2, n}$ for $n$ odd is given by

$$
\psi_{\lambda}^{I_{2, n}}(q, t)= \begin{cases}(-1)^{\frac{\lambda}{2}} t^{\frac{(n+2) \lambda}{2}} q^{\frac{(n+2)}{2} \frac{\lambda}{2}\left(\frac{\lambda}{2}-1\right)}\left(\frac{q}{t}\right)^{\frac{\lambda}{2} \frac{(n+1)}{2}} N_{\lambda} \frac{(t ; q)_{\lambda / 2}}{(q ; q)_{\lambda / 2}} & \lambda \text { even } \\ 0 & \lambda \text { odd } .\end{cases}
$$

We will provide some evidences of this proposal in the following subsection.

Even $\boldsymbol{n}$. The wave function for the $I_{2,2 n}$ is proposed by [30] as (up to normalization)

$$
\psi_{\lambda}^{I_{2,2 n-2}}(x)=N_{\lambda} \frac{t^{n \lambda / 2} q^{n \lambda^{2} / 4}}{(t ; q)} \sum_{m=0}^{\lambda} \frac{(t ; q)_{m}(t ; q)_{\lambda-m}}{(q ; q)_{m}(q ; q)_{\lambda-m}} q^{-n\left(\frac{\lambda}{2}-m\right)^{2}} x^{2 m-\lambda} .
$$

As in the previous sections, we can obtain $I_{2,0}$ by integrating the wave function for the regular puncture with vector and hypermultiplet kernel

$$
\psi_{\lambda}^{I_{2,0}}(a ; q, t)=\oint[d z] I_{\mathrm{vec}}(z) I_{\mathrm{hyp}}(z, a) \psi_{\lambda}(z)=\oint \frac{d z}{2 \pi i z} \frac{\left(1-z^{ \pm 2}\right)}{2} \frac{\left(q z^{ \pm 2,0} ; q\right)}{\left(t^{\frac{1}{2}} z^{ \pm} a^{ \pm} ; q\right)} \underline{P}_{\lambda}(z ; q, t) .
$$

We have verified that this expression agrees with (4.7) for $n=1$ up to high orders in $q$.

\subsection{Examples}

In this section, we use (4.6) to compute the Macdonald index for a number of examples and provide consistency checks. 


\subsubsection{Consistency checks}

Let us first consider a number of examples where we can cross-check the conjectured formula (4.6) against independent computations.

SU(2) SYM. The pure YM theory can be realized by a pair of $I_{2,-1}$ punctures on a sphere. From the TQFT structure of the index, we write

$$
\begin{aligned}
I_{\mathrm{SYM}}= & \sum_{\lambda} \psi_{\lambda}^{I_{2,-1}} \psi_{\lambda}^{I_{2,-1}}=\sum_{n \geq 0} N_{2 n}^{2} \frac{(t ; q)_{n}^{2}}{(q ; q)_{n}^{2}} t^{2 n} q^{n(n-1)} \\
= & 1+q^{2} T+q^{3}\left(T-T^{3}\right)+q^{4}\left(T-T^{3}\right)+q^{5}\left(T-T^{3}\right)+q^{6}\left(-T^{3}+T^{2}+T\right) \\
& +q^{7}\left(-T^{4}-T^{3}+T^{2}+T\right)+O\left(q^{8}\right),
\end{aligned}
$$

where $t=q T$. This result indeed agrees with the direct computation.

$\left(\boldsymbol{A}_{1}, \boldsymbol{A}_{\mathbf{0}}\right)$ theory. This should describe a theory with no massless degrees of freedom. We indeed find

$$
I_{\left(A_{1}, A_{0}\right)}=\sum_{\lambda} C_{\lambda}^{-1} \psi_{\lambda}^{I_{2,1}}(q, t)=1
$$

to high orders in $q$. This is rather a non-trivial check of the proposal (4.6), since each term in the sum has to cancel exactly up on summing over all terms.

$\left(\boldsymbol{A}_{\mathbf{1}}, \boldsymbol{A}_{\mathbf{3}}\right)=\left(\boldsymbol{A}_{\mathbf{1}}, \boldsymbol{D}_{\mathbf{3}}\right)$ theory. The $\left(A_{1}, A_{3}\right)$ theory is isomorphic to $\left(A_{1}, D_{3}\right)$ theory. The former description can be obtained from a single $I_{2,4}$ puncture and the latter description can be obtained from $I_{2,1}$ and a regular puncture. We find that these two descriptions indeed give us the same index

$$
I_{\left(A_{1}, A_{3}\right)}(x)=\sum_{\lambda} C_{\lambda}^{-1} \psi_{\lambda}^{I_{2,4}}(x)=\sum_{\lambda} \psi_{\lambda}^{I_{2,1}} \psi_{\lambda}(a)=I_{\left(A_{1}, D_{3}\right)}(a),
$$

upon identifying $x=a^{2}$. This result provides a consistency check between (4.6) and (4.7).

We find that the Schur limit of this index can be written in a very simple form

$$
I_{\left(A_{1}, D_{3}\right)}(a)=\mathrm{PE}\left[\frac{q-q^{3}}{(1-q)\left(1-q^{3}\right)} \chi_{\text {adj }}(a)\right] .
$$

The first term inside the PE is coming from the conserved current multiplet.

\subsubsection{Conjecture for the Macdonald indices of Argyres-Douglas theories}

$\left(\boldsymbol{A}_{\mathbf{1}}, \boldsymbol{A}_{\mathbf{2}}\right)$ theory. It can be obtained from $I_{2,3}$ punctured sphere. We conjecture its Macdonald index is given as

$$
\begin{aligned}
I_{\left(A_{1}, A_{2}\right)}=\sum_{\lambda} C_{\lambda}^{-1} \psi_{\lambda}^{I_{2,3}}= & 1+q^{2} T+q^{3} T+q^{4} T+q^{5} T+q^{6}\left(T^{2}+T\right)+q^{7}\left(T^{2}+T\right) \\
& +q^{8}\left(2 T^{2}+T\right)+q^{9}\left(2 T^{2}+T\right)+q^{10}\left(3 T^{2}+T\right)+O\left(q^{11}\right),
\end{aligned}
$$

where $t=q T$. This theory does not have a Higgs branch. This can be seen from triviality of the Hall-Littlewood limit of the index $q \rightarrow 0$. 
We find that this expression can be also written as

$$
I_{\left(A_{1}, A_{2}\right)}=\mathrm{PE}\left[\frac{q^{2} T-q^{4} T^{2}}{(1-q)\left(1-q^{5} T^{2}\right)}+O\left(q^{11}\right)\right],
$$

where the $O\left(q^{11}\right)$ terms vanish in the limit $T \rightarrow 1$. The first term inside the PE is coming from the short multiplet $\hat{\mathcal{C}}_{0(0,0)}$ (and their powers) using the notation of [58]. See also appendix $\mathrm{B}$ of [7]. This is the multiplet containing the stress-energy tensor. The Macdonald index for the short multiplet $\hat{\mathcal{C}}_{R\left(j_{1}, j_{2}\right)}$ is given as

$$
I_{\hat{\mathcal{C}}_{R\left(j_{1}, j_{2}\right)}}=(-1)^{2\left(j_{1}+j_{2}\right)} \frac{q^{2 j_{1}+1} t^{R+1+j_{2}-j_{1}}}{1-q}=(-1)^{2\left(j_{1}+j_{2}\right)} \frac{q^{R+2+j_{1}+j_{2}} T^{R+1+j_{2}-j_{1}}}{1-q} .
$$

We see that the stress-energy tensor multiplet contributes $\frac{q^{2} T}{1-q}$ to the Macdonald index.

Since any SCFT has a stress-energy tensor multiplet, the operator appear in the OPE of it should be also present in the theory. The OPE of the stress-energy tensor multiplet $\hat{\mathcal{C}}_{0(0,0)} \times \hat{\mathcal{C}}_{0(0,0)}$ contains $[59]^{5}$

$$
\hat{\mathcal{C}}_{0(0,0)} \times \hat{\mathcal{C}}_{0(0,0)} \sim \hat{\mathcal{C}}_{0\left(\frac{\ell}{2}, \frac{\ell}{2}\right)}+\hat{\mathcal{C}}_{1\left(\frac{\ell}{2}, \frac{\ell}{2}\right)}+\cdots,
$$

where we have only written short multiplets appear in the OPE that contributes to the Macdonald index. The $\hat{\mathcal{C}}_{0\left(\frac{\ell}{2}, \frac{\ell}{2}\right)}$ multiplets are higher-spin conserved currents which have to be absent unless the theory is free or has a decoupled sector [60]. This multiplet contributes to the index by $\frac{q^{\ell+2} T}{1-q}$. Indeed, we see from the index (4.13) that there is no $\hat{\mathcal{C}}_{0\left(\frac{\ell}{2}, \frac{\ell}{2}\right)}$ multiplet other than $\ell=0$, which contains the stress-energy tensor.

Among the terms appear on the r.h.s. of the OPE (4.16), $\hat{\mathcal{C}}_{1\left(\frac{\ell}{2}, \frac{\ell}{2}\right)}$ multiplet contributes $\frac{q^{\ell+3} T^{2}}{1-q}$ to the index. Since the index has coefficient 0 for the $q^{4} T^{2}$ term, $\hat{\mathcal{C}}_{1\left(\frac{1}{2}, \frac{1}{2}\right)}$ cannot be present. We also see $\hat{\mathcal{C}}_{1\left(\frac{\ell}{2}, \frac{\ell}{2}\right)}$ with even $\ell$ is absent. Our result agrees with the analysis of [59] where they show that $\hat{\mathcal{C}}_{1\left(\frac{1}{2}, \frac{1}{2}\right)}$ is absent for the theory with central charge $c=\frac{11}{30}$ which is the value of $\left(A_{1}, A_{2}\right)$ theory [61]. See also [62].

$\left(\boldsymbol{A}_{1}, \boldsymbol{A}_{4}\right)$ theory. It can be obtained from $I_{2,5}$ punctured sphere. Our conjectured Macdonald index is

$$
\begin{aligned}
I_{\left(A_{1}, A_{4}\right)}=\sum_{\lambda} C_{\lambda}^{-1} \psi_{\lambda}^{I_{2,5}}= & 1+q^{2} T+q^{3} T+q^{4}\left(T^{2}+T\right)+q^{5}\left(T^{2}+T\right)+q^{6}\left(2 T^{2}+T\right) \\
& +q^{7}\left(2 T^{2}+T\right)+q^{8}\left(T^{3}+3 T^{2}+T\right)+O\left(q^{9}\right) .
\end{aligned}
$$

It also reduces to 1 in the Hall-Littlewood limit $q \rightarrow 0$ as expected.

We find the index can be written in terms of a Plethystic exponential as

$$
I_{\left(A_{1}, A_{4}\right)}=\mathrm{PE}\left[\frac{q^{2} T-q^{6} T^{3}}{(1-q)\left(1-q^{7} T^{3}\right)}+O\left(q^{15}\right)\right]
$$

where $O\left(q^{15}\right)$ term vanishes as $T \rightarrow 1$.

\footnotetext{
${ }^{5}$ The author would like to thank Wenbin Yan for discussions on this point.
} 
Here we see that some of the short-multiplets appear in the OPE of the 3 stress-energy tensor multiplets

$$
\hat{\mathcal{C}}_{0(0,0)} \times \hat{\mathcal{C}}_{0(0,0)} \times \hat{\mathcal{C}}_{0(0,0)},
$$

should be absent, because there is no term of the form $q^{6} T^{3}$ in the index. Among the operators appear in the OPE of 3 stress-energy tensors, the multiplet contributing $\frac{q^{6} T^{3}}{1-q}$ has to be absent. The natural candidate would be $\hat{\mathcal{C}}_{2(1,1)}$, but we cannot rule out other possibilities from the index before working out the selection rule, because any $\hat{\mathcal{C}}_{R\left(1, j_{2}\right)}$ with $R+j_{2}=3$ for an integer $j_{2}$ will give the same index.

$\left(\boldsymbol{A}_{\mathbf{1}}, \boldsymbol{A}_{\mathbf{2 n}}\right)$ theory. We put $I_{2,2 n+1}$ puncture on a sphere. We conjecture the Macdonald index to be of the form

$$
I_{\left(A_{1}, A_{2 n}\right)}=\sum_{\lambda} C_{\lambda}^{-1} \psi_{\lambda}^{I_{2,2 n+1}}=\operatorname{PE}\left[\frac{q^{2} T-\left(q^{2} T\right)^{n+1}}{(1-q)\left(1-q^{2 n+3} T^{n+1}\right)}+\cdots\right],
$$

where omitted piece vanishes in the Schur limit $T \rightarrow 1$.

There is no $\left(q^{2} T\right)^{n+1}$ term in the index. Therefore the short multiplet that appear in the OPE of $\left(\hat{\mathcal{C}}_{0(0,0)}\right)^{n+1}$ that contributes to the index as $\frac{\left(q^{2} T\right)^{n+1}}{1-q}$ is absent. The short multiplet $\hat{\mathcal{C}}_{n\left(\frac{n}{2}, \frac{n}{2}\right)}$ contributes the same amount so that it might be absent.

$\left(\boldsymbol{A}_{\mathbf{1}}, \boldsymbol{D}_{\mathbf{5}}\right)$ theory. It can be obtained from a sphere with a $I_{2,3}$ puncture and a regular puncture. We get

$$
\begin{aligned}
I_{\left(A_{1}, D_{5}\right)}(a)= & \sum_{\lambda} \psi_{\lambda}^{I_{2,3}} \psi_{\lambda}(a) \\
= & 1+q T \chi_{3}+q^{2}\left(T\left(\chi_{3}+\chi_{1}\right)+T^{2} \chi_{5}\right)+q^{3}\left(T\left(\chi_{3}+\chi_{1}\right)+T^{2}\left(\chi_{5}+2 \chi_{3}\right)+T^{3} \chi_{7}\right) \\
& +q^{4}\left(T\left(\chi_{3}+\chi_{1}\right)+T^{2}\left(2 \chi_{5}+3 \chi_{3}+2 \chi_{1}\right)+T^{3}\left(\chi_{7}+2 \chi_{5}\right)+T^{4} \chi_{9}\right)+O\left(q^{5}\right),
\end{aligned}
$$

where $\chi_{n}$ denotes the character for the $n$-dimensional representation of $\mathrm{SU}(2)$. When we take the Hall-Littlewood limit $q \rightarrow 0$ with $t$ fixed, we get

$$
I_{\left(A_{1}, D_{5}\right)}(a)=\sum_{n \geq 0} \chi_{2 n+1}(a) t^{n}=\frac{1-t^{2}}{\left(1-t a^{2}\right)\left(1-t a^{-2}\right)(1-t)},
$$

which is the same as the HL index of the $\left(A_{1}, A_{3}\right)$ theory given in (3.6) with $n=2$ and $w=a^{2}$. This is nothing but the Hilbert series of $\mathbb{C}^{2} / \mathbb{Z}_{2}$.

The first term of the index (4.21) comes from the conserved current of the $\mathrm{SU}(2)$ flavor symmetry. We find that the index has the form

$$
I_{\left(A_{1}, D_{5}\right)}=\mathrm{PE}\left[\frac{q T}{1-q} \chi_{\mathrm{adj}}(a)+\ldots\right],
$$

where the omitted term vanishes in the Schur limit.

\section{$5 \mathcal{N}=1$ class $\mathcal{S}$ theories}

For every theories in $\mathcal{N}=1$ class $\mathcal{S}$, the superconformal indices can be written in terms of the correlation functions of a (generalized) topological field theory on the UV curve [38]. See also $[20,63-65]$. In this section, we generalize our discussions to the $\mathcal{N}=1$ case. 


\subsection{Mixed Schur index}

The $\mathcal{N}=1$ index for the class $\mathcal{S}$ is defined as

$$
I(p, q, \xi ; \boldsymbol{x})=\operatorname{Tr}(-1)^{F} \mathfrak{p}^{j_{1}+j_{2}+\frac{R_{0}}{2}} \mathfrak{q}^{j_{2}-j_{1}+\frac{R_{0}}{2}} \xi^{\mathcal{F}} \prod_{i} x_{i}^{F_{i}},
$$

where $R_{0}$ is the UV R-charge and $\mathcal{F}$ is the global U(1) charge conserved for a generic class $\mathcal{S}$ theory. One of the simplification limit of the above index is to take $\xi=\sqrt{\mathfrak{q} / \mathfrak{p}}$, called the mixed Schur limit [38]. It is given as

$$
I(\mathfrak{p}, \mathfrak{q} ; \boldsymbol{x})=\operatorname{Tr}(-1)^{F} \mathfrak{p}^{j_{1}+j_{2}+J_{-}} \mathfrak{q}^{j_{2}-j_{1}+J_{+}} \prod_{i} x_{i}^{F_{i}},
$$

where we used $J_{ \pm}=\frac{1}{2}\left(R_{0} \pm \mathcal{F}\right)$. In this limit, the chiral multiplet contribution of the index can be written as

$$
I_{\text {chi }}(\mathfrak{p}, \mathfrak{q} ; \boldsymbol{z})=\operatorname{PE}\left[\frac{\mathfrak{p}^{\frac{J_{-}}{2}} \mathfrak{q}^{\frac{J_{+}}{2}} \chi_{\Lambda}(\boldsymbol{z})-\mathfrak{p}^{1-\frac{J_{-}}{2}} \mathfrak{q}^{1-\frac{J_{+}}{2}} \chi_{\bar{\Lambda}}(\boldsymbol{z})}{(1-\mathfrak{p})(1-\mathfrak{q})}\right],
$$

where $\chi_{\Lambda}$ is the character of the representation $\Lambda$ of the gauge group. In the mixed Schur limit, the hypermultiplets charged with $\left(J_{+}, J_{-}\right)=(1,0)$ gives the index purely a function of $\mathfrak{q}$ as

$$
I_{\text {hyp }}^{(1,0)}(\mathfrak{p}, \mathfrak{q} ; \boldsymbol{z})=\operatorname{PE}\left[\frac{\mathfrak{q}^{\frac{1}{2}}}{1-\mathfrak{q}}\left(\chi_{\Lambda}(\boldsymbol{z})+\chi_{\bar{\Lambda}}(\boldsymbol{z})\right)\right],
$$

and the hypermultiplets with $\left(J_{+}, J_{-}\right)=(0,1)$ gives

$$
I_{\text {hyp }}^{(0,1)}(\mathfrak{p}, \mathfrak{q} ; \boldsymbol{z})=\operatorname{PE}\left[\frac{\mathfrak{p}^{\frac{1}{2}}}{1-\mathfrak{p}}\left(\chi_{\Lambda}(\boldsymbol{z})+\chi_{\bar{\Lambda}}(\boldsymbol{z})\right)\right] .
$$

For the chiral multiplets with $\left(J_{+}, J_{-}\right)=(0,2)$ in the adjoint representation of $G$, we get

$$
I_{\text {chi }}^{(0,2)}(\mathfrak{p}, \mathfrak{q} ; \boldsymbol{z})=\operatorname{PE}\left[\left(\frac{\mathfrak{p}}{1-\mathfrak{p}}-\frac{\mathfrak{q}}{1-\mathfrak{q}}\right) \chi_{\mathrm{adj}}(\boldsymbol{z})\right]
$$

where $\chi_{R}$ is the character of the representation $R$ of $G$.

The index of the theory in class $\mathcal{S}$ corresponding to the UV curve $\mathcal{C}_{g, n}$ with normal bundle degrees $(p, q)$ is given by

$$
I\left(\boldsymbol{a}_{\boldsymbol{i}} ; \mathfrak{p}, \mathfrak{q}, \xi\right)=\sum_{\boldsymbol{\lambda}}\left(C_{\boldsymbol{\lambda}}^{+}\right)^{p}\left(C_{\boldsymbol{\lambda}}^{-}\right)^{q} \prod_{i=1}^{n} \psi_{\boldsymbol{\lambda}}^{\sigma_{i}}\left(\boldsymbol{a}_{\boldsymbol{i}}\right),
$$

where $\boldsymbol{\lambda}$ labels the representations of $\Gamma$ and $\psi_{\boldsymbol{\lambda}}^{\sigma}(\boldsymbol{z})$ is the wave function associated to the puncture of color $\sigma= \pm$. The wave function in the mixed Schur limit becomes

$$
\psi_{\boldsymbol{\lambda}}^{+}(\boldsymbol{z})=\operatorname{PE}\left[\frac{\mathfrak{q}}{1-\mathfrak{q}} \chi_{\mathrm{adj}}(\boldsymbol{z})\right] \chi_{\boldsymbol{\lambda}}(\boldsymbol{z}), \quad \psi_{\boldsymbol{\lambda}}^{-}(\boldsymbol{z})=\operatorname{PE}\left[\frac{\mathfrak{p}}{1-\mathfrak{p}} \chi_{\mathrm{adj}}(\boldsymbol{z})\right] \chi_{\boldsymbol{\lambda}}(\boldsymbol{z}) .
$$


The wave functions for the irregular punctures with color $\sigma= \pm$ are given by simply choosing the $\mathcal{N}=2$ wave function with different arguments $\psi_{\boldsymbol{\lambda}}^{I_{k, n},+}=\psi_{\boldsymbol{\lambda}}^{I_{k, n}}(\mathfrak{p})$ and $\psi_{\boldsymbol{\lambda}}^{I_{k, n},-}=$ $\psi_{\boldsymbol{\lambda}}^{I_{k, n}}(\mathfrak{q})$. In this limit, the structure constant can be also simply written as

$$
C_{\boldsymbol{\lambda}}^{+}=C_{\boldsymbol{\lambda}}(\mathfrak{q}) \quad C_{\boldsymbol{\lambda}}^{-}=C_{\boldsymbol{\lambda}}(\mathfrak{p})
$$

where $C_{\boldsymbol{\lambda}}(\mathfrak{q})$ is the structure constant for $\mathcal{N}=2$ theory.

Note that one can flip the color of the wave function by attaching a chiral multiplet transforming under the adjoint of the flavor symmetry:

$$
\psi_{\boldsymbol{\lambda}}^{-}(\boldsymbol{z})=I_{\text {chi }}^{(0,2)}(\boldsymbol{z}) \psi_{\boldsymbol{\lambda}}^{+}(\boldsymbol{z}), \quad \psi_{\boldsymbol{\lambda}}^{+}(\boldsymbol{z})=I_{\text {chi }}^{(2,0)}(\boldsymbol{z}) \psi_{\boldsymbol{\lambda}}^{-}(\boldsymbol{z})
$$

This explains why we attach flavor adjoint chiral multiplets to the oppositely colored punctures.

Equipped with the wave functions for the irregular punctures, we can easily compute the index in the mixed Schur limit. Note that even when the $\mathcal{N}=2$ counterpart is nonconformal, $\mathcal{N}=1$ version can actually flow to a SCFT in certain cases. For example, $\mathrm{SU}(3)$ theory with $N_{f}=5$ is non-conformal for $\mathcal{N}=2$, but it is in the conformal window for $\mathcal{N}=1$ theory. We can indeed compute the indices for these cases using the result of section 2. In section 5.2, we consider simplest examples.

\section{$5.2 \mathrm{SU}(2) \mathrm{SYM}$ with $N_{f}=0,1,2,3$}

In this section, we verify that the mixed Schur index is indeed reproduced by the generalized TQFT we discussed.

Pure YM. Let us compute the index naively by using the UV matter content. Here we only have a vector multiplet. We get [66]

$$
\begin{aligned}
I_{\mathrm{YM}} & =(\mathfrak{p} ; \mathfrak{p})(\mathfrak{q} ; \mathfrak{q}) \oint \frac{d z}{2 \pi i z} \Delta(z)\left(\mathfrak{q} z^{ \pm 2} ; \mathfrak{q}\right)\left(\mathfrak{p} z^{ \pm 2} ; \mathfrak{q}\right)=\frac{1}{2} \sum_{m, n \in \mathbb{Z}} \mathfrak{p}^{\frac{1}{2} m(m+1)} \mathfrak{q}^{\frac{1}{2} n(n+1)} \oint \frac{d z}{2 \pi i z} z^{2(m-n)} \\
& =\sum_{m \in \mathbb{Z} \geq 0}(\mathfrak{p q})^{\frac{1}{2} m(m+1)}=\sum_{\lambda} \psi_{\lambda}^{I_{2,-1,+}} \psi_{\lambda}^{I_{2,-1},-}
\end{aligned}
$$

where we used the Jacobi triple product identity. We indeed get the mixed Schur index from the TQFT.

SQCD with $\boldsymbol{N}_{\boldsymbol{f}}=\mathbf{1}$. This theory has a dynamically generated runaway superpotential, therefore we cannot define proper superconformal index. Nevertheless, we compute the index at the UV fixed point with incorrect R-charges for the chiral multiplets. Namely, we pick $R=\frac{1}{2}$ for the chiral multiplets. This value is the correct R-charge for the massdeformed $\mathcal{N}=2$ SCFTs such as $\mathrm{SU}(N)$ theory with $2 N$ flavors. 
Now, we apply the integral formula for the index to compute

$$
\begin{aligned}
I_{N_{f}=1}(\mathfrak{p}, \mathfrak{q} ; a)= & (\mathfrak{p} ; \mathfrak{p})(\mathfrak{q} ; \mathfrak{q}) \oint \frac{d z}{2 \pi i z} \Delta(z) \frac{\left(\mathfrak{q} z^{ \pm 2} ; \mathfrak{q}\right)\left(\mathfrak{p} z^{ \pm 2} ; \mathfrak{q}\right)}{\left(\mathfrak{q}^{\frac{1}{2}} z^{ \pm} a^{ \pm} ; \mathfrak{q}\right)} \\
= & 1+\frac{t}{y}+t^{2}\left(-a^{2}-\frac{1}{a^{2}}+\frac{2}{y^{2}}\right)+t^{3}\left(-\frac{a^{2}}{y}-\frac{1}{a^{2} y}+\frac{3}{y^{3}}-\frac{1}{y}\right) \\
& +t^{4}\left(-\frac{2 a^{2}}{y^{2}}-\frac{2}{a^{2} y^{2}}+\frac{5}{y^{4}}-\frac{1}{y^{2}}\right)+O\left(t^{5}\right),
\end{aligned}
$$

where $\mathfrak{p}=t y, \mathfrak{q}=t / y$. This result agrees with the TQFT on a sphere with one irregular puncture $I_{2 .-1}$ and one regular puncture with $(p, q)=(0,0)$, which is given by

$$
I_{\mathrm{TQFT}}(\mathfrak{p}, \mathfrak{q} ; a)=\sum_{\lambda} \psi_{\lambda}^{I_{2,-1},+} \psi_{\lambda}^{-}(a)=\frac{1}{\left(\mathfrak{q} a^{ \pm 2,0} ; \mathfrak{q}\right)} \sum_{m \in \mathbb{Z}_{\geq 0}}(-1)^{m} \mathfrak{p}^{\frac{1}{2} m(m+1)} \chi_{R_{m}}(a) .
$$

Note that we have only kept the diagonal subgroup of the full flavor symmetry $\mathrm{U}(1)_{L} \times \mathrm{U}(1)_{R}$.

SQCD with $\boldsymbol{N}_{\boldsymbol{f}}=\mathbf{2}$. This theory without superpotential confines with a deformed moduli space, but we perform the computation with the same philosophy as before. Let us take the (wrong) R-charge $1 / 2$ to compute the index. We have two different ways to construct the theory, as we have discussed in the case of $\mathcal{N}=2$ counterpart. Here depending on the choice of the colors on the punctures we actually get different indices because these choices determine the superpotential that are allowed [67].

Let us first consider the case with two irregular punctures $I_{2,0}$ of each color. This configuration realizes the theory with a quartic superpotential between two quarks. We write the index as

$$
I_{N_{f}=1+1}(\mathfrak{p}, \mathfrak{q} ; a, b)=(\mathfrak{p} ; \mathfrak{p})(\mathfrak{q} ; \mathfrak{q}) \oint \frac{d z}{2 \pi i z} \Delta(z) \frac{\left(\mathfrak{q} z^{ \pm 2} ; \mathfrak{q}\right)\left(\mathfrak{p} z^{ \pm 2} ; \mathfrak{q}\right)}{\left(\mathfrak{q}^{\frac{1}{2}} z^{ \pm} a^{ \pm} ; \mathfrak{q}\right)\left(\mathfrak{p}^{\frac{1}{2}} z^{ \pm} b^{ \pm} ; \mathfrak{p}\right)},
$$

which agrees with the TQFT expression

$$
I_{\mathrm{TQFT}}(\mathfrak{p}, \mathfrak{q} ; a, b)=\sum_{\lambda} \psi_{\lambda}^{I_{2,0},+}(a) \psi_{\lambda}^{I_{2,0},-}(b)
$$

Now, let us consider a 3-punctured sphere realization of $N_{f}=2$ theory. We have two regular + punctures, and one irregular $I_{2,-1}$ with - color. We pick the normal bundle degrees to be $(1,0)$. This realizes the $N_{f}=2$ theory without quartic superpotential, which gives the index to be

$$
I_{N_{f}=2+0}(\mathfrak{p}, \mathfrak{q} ; a, b)=(\mathfrak{p} ; \mathfrak{p})(\mathfrak{q} ; \mathfrak{q}) \oint \frac{d z}{2 \pi i z} \Delta(z) \frac{\left(\mathfrak{q} z^{ \pm 2} ; \mathfrak{q}\right)\left(\mathfrak{p} z^{ \pm 2} ; \mathfrak{q}\right)}{\left(\mathfrak{q}^{\frac{1}{2}} z^{ \pm} a^{ \pm} ; \mathfrak{q}\right)\left(\mathfrak{q}^{\frac{1}{2}} z^{ \pm} b^{ \pm} ; \mathfrak{q}\right)} .
$$

It agrees with the TQFT expression

$$
I_{\mathrm{TQFT}}(\mathfrak{p}, \mathfrak{q} ; x, y)=\sum_{\lambda} C_{\lambda}^{-} \psi_{\lambda}^{I_{2,-1},-} \psi_{\lambda}^{+}(x) \psi_{\lambda}^{+}(y),
$$

upon identifying $a=x y, b=x / y$. 
SQCD with $N_{f}=3$. This theory can be realized by a sphere with two regular punctures of + color and one irregular puncture $I_{2,0}$ with - color, and normal bundle degrees $(p, q)=$ $(1,0)$. It splits 3 flavors into $2+1$ with a quartic superpotential interaction. The index can be written as

$$
I_{N_{f}=2+1}(\mathfrak{p}, \mathfrak{q} ; a, b, c)=(\mathfrak{p} ; \mathfrak{p})(\mathfrak{q} ; \mathfrak{q}) \oint \frac{d z}{2 \pi i z} \Delta(z) \frac{\left(\mathfrak{q} z^{ \pm 2} ; \mathfrak{q}\right)\left(\mathfrak{p} z^{ \pm 2} ; \mathfrak{q}\right)}{\left(\mathfrak{q}^{\frac{1}{2}} z^{ \pm} a^{ \pm} ; \mathfrak{q}\right)\left(\mathfrak{q}^{\frac{1}{2}} z^{ \pm} b^{ \pm} ; \mathfrak{q}\right)\left(\mathfrak{p}^{\frac{1}{2}} z^{ \pm} c^{ \pm} ; \mathfrak{p}\right)}
$$

We find that it agrees with the TQFT expression

$$
I_{\mathrm{TQFT}}(\mathfrak{p}, \mathfrak{q} ; x, y)=\sum_{\lambda} C_{\lambda}^{-} \psi_{\lambda}^{+}(x) \psi_{\lambda}^{+}(y) \psi_{\lambda}^{I_{2,0},-}(c),
$$

upon identifying $a=x y, b=x / y$.

\section{Acknowledgments}

The author would like to thank Abhijit Gadde, Ken Intriligator, Yuji Tachikawa and Wenbin Yan for useful discussions and correspondence. The author is grateful for the hospitality of the Simons Center for Geometry and Physics during the 2015 Simons Workshop in Mathematics and Physics and also Korea Institute for Advanced Study. This work is supported by the US Department of Energy under UCSD's contract de-sc0009919.

Open Access. This article is distributed under the terms of the Creative Commons Attribution License (CC-BY 4.0), which permits any use, distribution and reproduction in any medium, provided the original author(s) and source are credited.

\section{References}

[1] D. Gaiotto, $N=2$ dualities, JHEP 08 (2012) 034 [arXiv:0904.2715] [INSPIRE].

[2] D. Gaiotto, G.W. Moore and A. Neitzke, Wall-crossing, Hitchin Systems and the WKB Approximation, arXiv:0907.3987 [INSPIRE].

[3] J. Kinney, J.M. Maldacena, S. Minwalla and S. Raju, An Index for 4 dimensional super conformal theories, Commun. Math. Phys. 275 (2007) 209 [hep-th/0510251] [INSPIRE].

[4] C. Romelsberger, Counting chiral primaries in $N=1, D=4$ superconformal field theories, Nucl. Phys. B 747 (2006) 329 [hep-th/0510060] [INSPIRE].

[5] A. Gadde, E. Pomoni, L. Rastelli and S.S. Razamat, S-duality and 2d Topological QFT, JHEP 03 (2010) 032 [arXiv:0910.2225] [INSPIRE].

[6] A. Gadde, L. Rastelli, S.S. Razamat and W. Yan, The 4d Superconformal Index from q-deformed $2 d$ Yang-Mills, Phys. Rev. Lett. 106 (2011) 241602 [arXiv:1104.3850] [INSPIRE].

[7] A. Gadde, L. Rastelli, S.S. Razamat and W. Yan, Gauge Theories and Macdonald Polynomials, Commun. Math. Phys. 319 (2013) 147 [arXiv:1110.3740] [InSPIRE].

[8] D. Gaiotto, L. Rastelli and S.S. Razamat, Bootstrapping the superconformal index with surface defects, JHEP 01 (2013) 022 [arXiv:1207.3577] [INSPIRE]. 
[9] L. Rastelli and S.S. Razamat, The superconformal index of theories of class $\mathcal{S}$, arXiv: 1412.7131 [INSPIRE].

[10] M. Aganagic, H. Ooguri, N. Saulina and C. Vafa, Black holes, q-deformed $2 d$ Yang-Mills and non-perturbative topological strings, Nucl. Phys. B 715 (2005) 304 [hep-th/0411280] [INSPIRE].

[11] T. Kawano and N. Matsumiya, 5D SYM on 3D Sphere and 2D YM, Phys. Lett. B 716 (2012) 450 [arXiv: 1206.5966] [INSPIRE].

[12] Y. Fukuda, T. Kawano and N. Matsumiya, $5 D S Y M$ and $2 D$ q-Deformed YM, Nucl. Phys. B 869 (2013) 493 [arXiv:1210.2855] [INSPIRE].

[13] T. Kawano and N. Matsumiya, 5D SYM on 3D Deformed Spheres, Nucl. Phys. B 898 (2015) 456 [arXiv: 1505.06565] [INSPIRE].

[14] F. Benini, T. Nishioka and M. Yamazaki, 4d Index to 3d Index and $2 d$ TQFT, Phys. Rev. D 86 (2012) 065015 [arXiv:1109.0283] [INSPIRE].

[15] L.F. Alday, M. Bullimore and M. Fluder, On S-duality of the Superconformal Index on Lens Spaces and $2 d$ TQFT, JHEP 05 (2013) 122 [arXiv:1301.7486] [INSPIRE].

[16] S.S. Razamat and M. Yamazaki, S-duality and the $N=2$ Lens Space Index, JHEP 10 (2013) 048 [arXiv: 1306.1543] [INSPIRE].

[17] N. Mekareeya, J. Song and Y. Tachikawa, 2d TQFT structure of the superconformal indices with outer-automorphism twists, JHEP 03 (2013) 171 [arXiv:1212.0545] [INSPIRE].

[18] M. Lemos, W. Peelaers and L. Rastelli, The superconformal index of class $S$ theories of type D, JHEP 05 (2014) 120 [arXiv:1212.1271] [INSPIRE].

[19] O. Chacaltana, J. Distler and A. Trimm, Tinkertoys for the Twisted D-Series, JHEP 04 (2015) 173 [arXiv: 1309.2299] [INSPIRE].

[20] P. Agarwal and J. Song, New $N=1$ Dualities from M5-branes and Outer-automorphism Twists, JHEP 03 (2014) 133 [arXiv:1311.2945] [INSPIRE].

[21] O. Chacaltana, J. Distler and A. Trimm, Tinkertoys for the $E_{6}$ theory, JHEP 09 (2015) 007 [arXiv: 1403.4604] [INSPIRE].

[22] O. Chacaltana, J. Distler and A. Trimm, Tinkertoys for the Twisted $E_{6}$ Theory, arXiv: 1501.00357 [INSPIRE].

[23] D. Xie, General Argyres-Douglas Theory, JHEP 01 (2013) 100 [arXiv:1204.2270] [InSPIRE].

[24] Y. Wang and D. Xie, Classification of Argyres-Douglas theories from M5 branes, arXiv: 1509.00847 [INSPIRE].

[25] P.C. Argyres and M.R. Douglas, New phenomena in SU(3) supersymmetric gauge theory, Nucl. Phys. B 448 (1995) 93 [hep-th/9505062] [InSPIRE].

[26] P.C. Argyres, M.R. Plesser, N. Seiberg and E. Witten, New $N=2$ superconformal field theories in four-dimensions, Nucl. Phys. B 461 (1996) 71 [hep-th/9511154] [INSPIRE].

[27] M. Buican and T. Nishinaka, On the superconformal index of Argyres-Douglas theories, J. Phys. A 49 (2016) 015401 [arXiv: 1505.05884] [INSPIRE].

[28] M. Buican, S. Giacomelli, T. Nishinaka and C. Papageorgakis, Argyres-Douglas Theories and S-duality, JHEP 02 (2015) 185 [arXiv:1411.6026] [INSPIRE]. 
[29] M. Buican and T. Nishinaka, Argyres-Douglas Theories, $S^{1}$ Reductions and Topological Symmetries, J. Phys. A 49 (2016) 045401 [arXiv:1505.06205] [InSPIRE].

[30] M. Buican and T. Nishinaka, Argyres-Douglas Theories, the Macdonald Index and an RG Inequality, arXiv:1509.05402 [INSPIRE].

[31] C. Beem, M. Lemos, P. Liendo, W. Peelaers, L. Rastelli and B.C. van Rees, Infinite Chiral Symmetry in Four Dimensions, Commun. Math. Phys. 336 (2015) 1359 [arXiv:1312.5344] [INSPIRE].

[32] C. Beem, W. Peelaers, L. Rastelli and B.C. van Rees, Chiral algebras of class S, JHEP 05 (2015) 020 [arXiv: 1408.6522] [INSPIRE].

[33] M. Lemos and W. Peelaers, Chiral Algebras for Trinion Theories, JHEP 02 (2015) 113 [arXiv: 1411.3252] [INSPIRE].

[34] C. Cordova and S.-H. Shao, Schur Indices, BPS Particles and Argyres-Douglas Theories, JHEP 01 (2016) 040 [arXiv: 1506.00265] [INSPIRE].

[35] S. Cecotti, A. Neitzke and C. Vafa, R-Twisting and 4d/2d Correspondences, arXiv:1006.3435 [INSPIRE].

[36] F. Benini, Y. Tachikawa and B. Wecht, Sicilian gauge theories and $N=1$ dualities, JHEP 01 (2010) 088 [arXiv:0909.1327] [INSPIRE].

[37] I. Bah, C. Beem, N. Bobev and B. Wecht, Four-Dimensional SCFTs from M5-Branes, JHEP 06 (2012) 005 [arXiv: 1203.0303] [INSPIRE].

[38] C. Beem and A. Gadde, The $N=1$ superconformal index for class $S$ fixed points, JHEP 04 (2014) 036 [arXiv:1212.1467] [InSPIRE].

[39] D. Xie, M5 brane and four dimensional $N=1$ theories I, JHEP 04 (2014) 154 [arXiv: 1307.5877] [INSPIRE].

[40] D. Xie and P. Zhao, Central charges and RG flow of strongly-coupled $N=2$ theory, JHEP 03 (2013) 006 [arXiv:1301.0210] [INSPIRE].

[41] D. Gaiotto, Asymptotically free $\mathcal{N}=2$ theories and irregular conformal blocks, J. Phys. Conf. Ser. 462 (2013) 012014 [arXiv:0908.0307] [INSPIRE].

[42] M. Taki, On AGT Conjecture for Pure Super Yang-Mills and W-algebra, JHEP 05 (2011) 038 [arXiv:0912.4789] [INSPIRE].

[43] C.A. Keller, N. Mekareeya, J. Song and Y. Tachikawa, The ABCDEFG of Instantons and $W$-algebras, JHEP 03 (2012) 045 [arXiv: 1111.5624] [INSPIRE].

[44] L.F. Alday, D. Gaiotto and Y. Tachikawa, Liouville Correlation Functions from Four-dimensional Gauge Theories, Lett. Math. Phys. 91 (2010) 167 [arXiv:0906.3219] [INSPIRE].

[45] N. Wyllard, A(N-1) conformal Toda field theory correlation functions from conformal $N=2$ $\mathrm{SU}(N)$ quiver gauge theories, JHEP 11 (2009) 002 [arXiv:0907.2189] [INSPIRE].

[46] G. Bonelli, K. Maruyoshi and A. Tanzini, Wild Quiver Gauge Theories, JHEP 02 (2012) 031 [arXiv:1112.1691] [INSPIRE].

[47] D. Gaiotto and J. Teschner, Irregular singularities in Liouville theory and Argyres-Douglas type gauge theories, I, JHEP 12 (2012) 050 [arXiv:1203.1052] [INSPIRE]. 
[48] H. Kanno, K. Maruyoshi, S. Shiba and M. Taki, $W_{3}$ irregular states and isolated $N=2$ superconformal field theories, JHEP 03 (2013) 147 [arXiv: 1301.0721] [INSPIRE].

[49] G. Andrews, A. Schilling and S. Warnaar, An $A_{2}$ Bailey lemma and Rogers-Ramanujan-type identities, J. Am. Math. Soc. 12 (1999) 677.

[50] P.C. Argyres, K. Maruyoshi and Y. Tachikawa, Quantum Higgs branches of isolated $N=2$ superconformal field theories, JHEP 10 (2012) 054 [arXiv: 1206.4700] [INSPIRE].

[51] K.A. Intriligator and N. Seiberg, Mirror symmetry in three-dimensional gauge theories, Phys. Lett. B 387 (1996) 513 [hep-th/9607207] [INSPIRE].

[52] P. Boalch, Irregular connections and Kac-Moody root systems, arXiv:0806.1050.

[53] S. Cremonesi, A. Hanany and A. Zaffaroni, Monopole operators and Hilbert series of Coulomb branches of $3 d \mathcal{N}=4$ gauge theories, JHEP 01 (2014) 005 [arXiv:1309.2657] [INSPIRE].

[54] S.S. Razamat and B. Willett, Down the rabbit hole with theories of class $\mathcal{S}$, JHEP 10 (2014) 99 [arXiv: 1403.6107] [INSPIRE].

[55] M. Del Zotto and A. Hanany, Complete Graphs, Hilbert Series and the Higgs branch of the $4 d \mathcal{N}=2\left(A_{n}, A_{m}\right) S C F T s$, Nucl. Phys. B 894 (2015) 439 [arXiv: 1403.6523] [INSPIRE].

[56] D. Gaiotto and E. Witten, S-duality of Boundary Conditions In $N=4$ Super Yang-Mills Theory, Adv. Theor. Math. Phys. 13 (2009) 721 [arXiv:0807.3720] [InSPIRE].

[57] K. Maruyoshi, C.Y. Park and W. Yan, BPS spectrum of Argyres-Douglas theory via spectral network, JHEP 12 (2013) 092 [arXiv: 1309.3050] [INSPIRE].

[58] F.A. Dolan and H. Osborn, On short and semi-short representations for four-dimensional superconformal symmetry, Annals Phys. 307 (2003) 41 [hep-th/0209056] [INSPIRE].

[59] P. Liendo, I. Ramirez and J. Seo, Stress-tensor OPE in $N=2$ Superconformal Theories, arXiv: 1509.00033 [INSPIRE].

[60] J. Maldacena and A. Zhiboedov, Constraining Conformal Field Theories with A Higher Spin Symmetry, J. Phys. A 46 (2013) 214011 [arXiv:1112.1016] [InSPIRE].

[61] A.D. Shapere and Y. Tachikawa, Central charges of $N=2$ superconformal field theories in four dimensions, JHEP 09 (2008) 109 [arXiv:0804.1957] [INSPIRE].

[62] M. Lemos and P. Liendo, Bootstrapping $\mathcal{N}=2$ chiral correlators, JHEP 01 (2016) 025 [arXiv: 1510.03866] [INSPIRE].

[63] A. Gadde, K. Maruyoshi, Y. Tachikawa and W. Yan, New N=1 Dualities, JHEP 06 (2013) 056 [arXiv: 1303.0836] [INSPIRE].

[64] P. Agarwal, I. Bah, K. Maruyoshi and J. Song, Quiver tails and $\mathcal{N}=1$ SCFTs from M5-branes, JHEP 03 (2015) 049 [arXiv: 1409.1908] [INSPIRE].

[65] P. Agarwal, K. Intriligator and J. Song, Infinitely many $\mathcal{N}=1$ dualities from $m+1-m=1$, JHEP 10 (2015) 035 [arXiv:1505.00255] [INSPIRE].

[66] V.P. Spiridonov and G.S. Vartanov, Vanishing superconformal indices and the chiral symmetry breaking, JHEP 06 (2014) 062 [arXiv: 1402.2312] [INSPIRE].

[67] D. Xie and K. Yonekura, Generalized Hitchin system, Spectral curve and $\mathcal{N}=1$ dynamics, JHEP 01 (2014) 001 [arXiv:1310.0467] [INSPIRE]. 